\title{
PDZ Scaffold Protein CAL Couples with Metabotropic Glutamate Receptor 5 to Protect Against Cell Apoptosis and Is a Potential Target in the Treatment of Parkinson's Disease
}

\author{
Wen Yuan Luo ${ }^{1}$ - Su Qian Xing ${ }^{1}$ - Ping Zhu ${ }^{1}$ • Chen Guang Zhang ${ }^{2} \cdot$ Hui Min Yang ${ }^{1}$ - Nicholas Van Halm-Lutterodt ${ }^{3,4}$. \\ $\mathrm{Li} \mathrm{Gu}{ }^{1} \cdot$ Hong Zhang ${ }^{1}$ (D)
}

Published online: 9 May 2019

(C) The American Society for Experimental NeuroTherapeutics, Inc. 2019

\begin{abstract}
Targeting mGluR5 has been an attractive strategy to modulate glutamate excitotoxicity for neuroprotection. Although human clinical trials using mGluR5 negative allosteric modulators (NAMs) have included some disappointments, recent investigations have added several more attractive small molecules to this field, providing a promise that the identification of more additional strategies to modulate mGluR5 activity might be potentially beneficial for the advancement of PD treatment. Here, we determined the role of the interacting partner CAL (cystic fibrosis transmembrane conductance regulator-associated ligand) in mGluR5-mediated protection in vitro and in vivo. In astroglial C6 cells, CAL deficiency blocked (S)-3, 5-dihydroxyphenylglycine (DHPG)-elicited p-AKT and p-ERK1/2, subsequently prevented group I mGluRs-mediated anti-apoptotic protection, which was blocked by receptor antagonist 1-aminoindan1, 5-dicarboxylic acid (AIDA), and PI3K or MEK inhibitor LY294002 or U0126. In rotenone-treated MN9D cells, both CAL and mGluR5 expressions were decreased in a time- and dose-dependent manner, and the correlation between these 2 proteins was confirmed by lentivirus-delivered CAL overexpression and knockdown. Moreover, CAL coupled with mGluR5 upregulated mGluR5 protein expression by inhibition of ubiquitin-proteasome-dependent degradation to suppress mGluR5-mediated p-JNK and to protect against cell apoptosis. Additionally, CAL also inhibited rotenone-induced glutamate release to modulate mGluR5 activity. Furthermore, in the rotenone-induced rat model of PD, AAV-delivered CAL overexpression attenuated behavioral deficits and dopaminergic neuronal death, while CAL deficiency aggravated rotenone toxicity. On the other hand, the protective effect of the mGluR5 antagonist MPEP was weakened by knocking down CAL. In vivo experiments also confirmed that CAL inhibited ubiquitinationproteasome-dependent degradation to modulate mGluR5 expression and JNK phosphorylation. Our findings show that CAL protects against cell apoptosis via modulating mGluR5 activity, and may be a new molecular target for an effective therapeutic strategy for PD.
\end{abstract}

Key Words mGluR5 $\cdot$ CAL $\cdot$ cell apoptosis $\cdot$ ubiquitination $\cdot$ Parkinson's disease

\section{Introduction}

Parkinson's disease (PD) is well acknowledged as a neurodegenerative disorder characterized by a gradual loss of

Wen Yuan Luo and Su Qian Xing contributed equally to this work.

\section{$\mathrm{Li} \mathrm{Gu}$}

guli75@ccmu.edu.cn

Hong Zhang

hzhang@ccmu.edu.cn

1 Department of Neurobiology, School of Basic Medical Sciences, Beijing Institute for Brain Disorders and Key Laboratory for Neurodegenerative Disorders of the Ministry of Education, Capital Medical University, Beijing 100069, China dopaminergic neurons involved in the nigrostriatal pathway, the cardinal symptoms of which include bradykinesia, resting tremor, rigidity, and postural instability [1]. Excessive glutamatergic transmission in the basal ganglia which induces

2 Department of Biochemistry and Molecular Biology, Capital Medical University, Beijing 100069, China

3 Department of Neurosurgery, Beijing Tiantan Hospital, Capital Medical University, Beijing 100069, China

4 Department of Orthopaedics and Neurosurgery, Keck Medical Center, University of Southern California, Los Angeles, CA 90033, USA 
excitotoxicity is also implicated in PD [2]. Glutamate responses are mediated by ionotropic glutamate receptors (iGluRs) and metabotropic glutamate receptors (mGluRs). mGluRs have been classified into 3 major groups according to their sequence homology and signal transduction profile: group I (mGluR1, mGluR5), group II (mGluR2, mGluR3), and group III (mGluR4, mGluR6, mGluR7, mGluR8) [3]. mGluR5 is highly expressed in the basal ganglia and localized postsynaptically to modulate glutamatergic transmission. Contrary to the activation of iGluRs that are generally responsible for fast excitatory synaptic transmission, mGluRs are implicated in the finetuning and/or circuit selective modulation of this excitatory transmission and has thus been proposed as a promising target for neuroprotection in PD [4-6]. Indeed, the blockade of mGluR5 with antagonist MPEP/MTEP has an anti-parkinsonian effect in animal models [7, 8]. Recent clinical trials support the therapeutic potential of certain mGluR5 negative allosteric modulators (NAMs) such as mavoglurant (AFQ056) and dipraglurant (ADX48621) [9, 10]. Although human clinical trials on using mGluR5 NAMs have showed some limitations, recent investigations have added several more attractive small molecules to this field [11], suggesting that precise modulation such as avoiding over activation or prolonged inhibition of mGluR5 might be required for therapeutic efficacy. Thus, the identification of more strategies that facilitate mGluR5 modulation might be potentially beneficial for PD treatment.

Although many factors are involved in the translation of data from basic science to clinical application, a common factor in pharmacological trials is the identification of highly specific regulators that target proteins of interest. It is possible that the therapeutic effects can be enhanced by selectively targeting the coupling of mGluR5 to its endogenous regulatory factors in PD subjects. Structurally, mGluR5 is comprised of a large extracellular $\mathrm{N}$-terminal domain, known as the Venus flytrap domain (VFD), which contains an orthosteric ligand-binding site, a hepta-helical transmembrane domain in which allosteric modulators positively and negatively affect glutamate activity bind, and the C-terminal intracellular domain for $\mathrm{G}$ protein coupling modulation that includes a PSD95/ Discslarge/ZO1 homology (PDZ)-binding motif [12]. PDZ domains play vital roles in organizing and maintaining complex scaffolding formations and are directly involved in the regulation of protein interactions with a range of cellular components for signaling pathways and further regulate the functions of these targeted proteins. Multiple PDZ proteins, such as Homer, NHERF-2, and Tamalin/GRASP, are found to associate with mGluR5 through the PDZ domain [13-15]. CAL (the cystic fibrosis transmembrane conductance regulator-associated ligand, also known as GOPC, PIST, or FIG) is a scaffold protein identified first as the interacting partner with the GTPase TC10 [16]. The structure contains 2 predicted coiled-coil domains and 1 PDZ domain, located primarily at the Golgi apparatus [17]. CAL has also been reported to interact with several more proteins such as the cystic fibrosis transmembrane conductance regulator (CFTR) $[18,19], \beta 1$-adrenergic receptor [20], somatostatin receptor subtype $5[21,22]$, and Cadherin 23 [23] to regulate these proteins' membrane expression and their intracellular trafficking. Moreover, CAL regulates claudin-1 and claudin-2 subcellular distribution to alter tight junction structure [24] and as a binding partner of Rhotekin to coordinate the maintenance of cellular polarity and synapses $[25,26]$. Furthermore, CAL is ubiquitously abundant in the nervous central system [27]. Animal studies show that a neuronal isoform of CAL that contains an 8 amino acid insertion in the second coiled-coil domain is involved in autophagy and neurodegeneration by interacting with $\delta 2$ glutamate receptor and autophagy regulator Beclin1 in lurcher mice [28, 29]. Additionally, the interaction of CAL with AMPA receptor (AMPAR) associates protein stargazin enhances AMPAR synaptic clustering in hippocampal neurons [30]. We have previously reported that CAL interacts with group I mGluRs (mGluR1 and mGluR5) at the C-terminus [31, 32]. To this end, it would be interesting to further investigate whether CAL plays any role in modulating mGluR5 activity to prevent neurotoxicity.

In the present study, we examined the effect of CAL on modulating the expression and activity of mGluR5 and further investigated its pathophysiological function in a rotenoneinduced PD model. We found that CAL protected against cell apoptosis by modulating mGluR5 protein expression as well as mGluR5-mediated AKT, ERK1/2, and JNK pathways to reduce glutamatergic hyperactivity. Our findings show that CAL is a new molecular target that modulates glutamate receptor activity, and may be a potential pharmacological strategy in PD therapy.

\section{Materials and Methods}

\section{Reagents}

1,4-Diamino-2, 3-dicyano-1,4-bis[2-aminophenylthio] butadiene (U0126, MEK inhibitor), 2-(4-morpholinyl)-8-phenyl4H-1-benzopyran-4-one hydrochloride (LY294002, PI3K inhibitor), (S)-3, 5-dihydroxyphenylglycine (DHPG, group I mGluRs agonist), (RS)-1-aminoindan-1,5-dicarboxylic acid (AIDA, group I antagonist), (s)-(+)- $\alpha$-amine-4-carboxy-2methylbenzene acetic acid (LY367385, mGluR1 antagonist), (RS)-2-chloro-5-hidroxyphenylglycine (CHPG, mGluR5 
agonist), and 2-methyl-6-(phenylethynyl) pyridine hydrochloride (MPEP, mGluR5 antagonist) were all from Tocris Biosciences (Ellisville, MO, USA). Rotenone, cycloheximide (CHX, protein synthesis inhibitor), MG132 (proteasome and calpain inhibitor), and staurosporine (STS, protein kinase inhibitor) were all purchased from Sigma-Aldrich (St. Louis, MO, USA). SP600125 was obtained from R\&D Systems (Minneapolis, MN, USA). U0126, LY294002, STS, rotenone, MG132, and MPEP were dissolved in dimethylsulfoxide (DMSO, Amresco Inc., Solon, Ohio, USA); DHPG was dissolved in water and CHPG, AIDA, and LY367385 were dissolved in $\mathrm{NaOH}$.

\section{Cell Culture and Treatment}

Mouse MN9D cells were generously provided by Professor Hui Yang (Capital Medical University, Beijing, China) and cultured in Dulbecco's modified Eagle's medium/Ham's F-12 (DMEM/F12; Corning, Manassas, VA, USA). Rat C6 astroglial cells were maintained in DMEM (Corning), supplemented with $10 \%$ fetal bovine serum (FBS) and $100 \mathrm{U} / \mathrm{ml}$ penicillin/streptomycin in a $37{ }^{\circ} \mathrm{C} /$ $5 \% \mathrm{CO}_{2}$ incubator. To induce the activity of group I mGluRs, C6 astroglial cells were treated with DHPG or CHPG. To block the activity of group I mGluRs, the cells were treated with AIDA, LY367385, or MPEP. U0126 or LY294002 was used to prevent activation of the ERK1/2 or AKT pathways. To inhibit protein synthesis, MN9D cell cultures were pretreated with CHX, and the proteasome activity was blocked by pretreatment with MG132. The JNK pathway was repressed by pretreatment with SP600125.

\section{Plasmids Construction and Transfection}

To modulate the level of CAL in $\mathrm{C} 6$ astroglial cells, short hairpin RNA (shRNA) duplexes directed against CAL were designed as follows: 5'-CACCGCATGTTGGA GAT GCTAT T C T T TCAA GA GAA GAATA GCAT CTCCAACATGCTTTTTTG-3' and 5'-GATC CAAAAAAGCATGTTGGAGATGCTATTCTTCTCTT GAAAGAATAGCATCTCCAACATGC-3'. Scramble control shRNA was as follows: 5'-CACCGTTCTCCGAA CGTGTCACGTCAAGAGATTACGTGACACGT TCGGAGAATTTTTTG-3' and 5'-GATCCAAAAAATTC TCCGAACGTGTCAGTAATC TCT TGACGTGA CACGTTCGGAGAAC-3'. Both of the shRNAs were synthesized and cloned into the plasmid pGPH1/GFP/ $\mathrm{Neo}$ at BamH1 and HindIII restriction sites (Shanghai Gene Pharma Co. Ltd., Shanghai, China), and abbreviated to scramble or CAL shRNA plasmid. To modulate the level of CAL in MN9D cells, the human full-length $C A L$ gene (NM_001017408.2) was cloned into the pAAV-
CMV-IRES-ZsGreen plasmid vector with Bam $\mathrm{H} 1$ and $E c o$ RI restriction sites for the overexpression of CAL (Likeligene, Beijing, China), and abbreviated to CMVIRES-GFP or CMV-IRES-hCAL-GFP. In addition, shRNAs that targeted the cds region of rat $C A L$ gene were cloned into the plasmids for knockdown of CAL (Viagene Biosciences, Shandong, China), and abbreviated to shCAL or shGFP. C6 astroglial cells or MN9D cells at $80 \%$ confluence were transfected with plasmids using Lipofectamine 3000 reagent (Invitrogen, Carlsbad, CA, USA) and then harvested and analyzed at $48 \mathrm{~h}$ after transfection.

\section{Cell Viability Using the MTT Assay}

MN9D cells were seeded into $96-$ well plates at $5 \times 10^{3}$ cells/ well. After $24 \mathrm{~h}$, cells at $80-90 \%$ confluence were treated with different drugs for the indicated time. 3-(4,5-Dimethylthiazol2-yl)-2, 5-diphenyltetrazolium bromide (MTT, $5 \mathrm{mg} / \mathrm{ml}$ ) was added to the cell cultures, and the plates were incubated for $4 \mathrm{~h}$ at $37{ }^{\circ} \mathrm{C}$. The formation of formazan was dissolved with DMSO $(150 \mu \mathrm{l})$ and the absorbance at $490 \mathrm{~nm}$ was determined using a microplate reader (Elx800; Bio-Tek Instruments, Winooski, VT, USA).

\section{Measurement of Cell Apoptosis by TUNEL Staining and Annexin-V/PI Staining}

Terminal deoxynucleotidyl transferase-mediated dUTP-biotin nick-end labeling (TUNEL) staining was performed using a TACS cell death detection kit (Roche Applied Science, Mannheim, Germany). Briefly, cells grown on glass coverslips were fixed with $4 \%$ paraformaldehyde (PFA) followed by permeabilization with $0.1 \%$ Triton $\mathrm{X}$ 100 and further processed for TUNEL staining. After washing 3 times with phosphate-buffered saline (PBS), Hoechst $33258(10 \mathrm{mg} / \mathrm{ml}$, Sigma-Aldrich) was added to counterstain the nuclei. Photomicrographs from at least 6 different locations on each coverslip were captured. Typically, 100-200 cells were analyzed to determine the number of TUNEL-positive (apoptotic) cells. Apoptotic cell numbers were presented as the percentage of TUNEL-positive cells in relation to total cell numbers. Annexin-V/PI staining was detected by FITC Annexin V Apoptosis Detection Kit 1 (BD pharmingen, San Diego, USA). Cells were washed twice with cold PBS and then resuspended in $1 \times$ binding buffer at a concentration of $\sim$ $1 \times 10^{6}$ cells $/ \mathrm{ml}$. After transfer $100 \mu$ of the solution $(1 \times$ $10^{5}$ cells) to a 5 -ml culture tube, $5 \mu$ of FITC Annexin V and $5 \mu \mathrm{l}$ PI were added into it. Gently vortexing the cells and incubating for $15 \mathrm{~min}$ at RT in the dark, $400 \mu \mathrm{l}$ of $1 \times$ binding buffer was added into each tube followed by analyzing by flow cytometry within $1 \mathrm{~h}$. 


\section{Lentivirus Construction and Recombinant Adeno-Associated Virus Generation}

Lentivirus construction was used in MN9D cells. For overexpression of CAL, the sequence of human $C A L$ cDNA was cloned into the pLVX-mCMV-ZsGreen lentivirus vector (Biowit Technologies, Shenzhen, China) with EcoRI and BamHI restriction sites (abbreviated to LV-CAL). The pLVX-mCMV-ZsGreen vector was used as the control (abbreviated to LV-NC). To knock down protein CAL, shRNA (5'GCTGAAGTGAAGCTGCTAA-3') target mouse $C A L$ (NM_001199272.1) or the negative control (5'-TCGC TTACCGATTCAGAATGG-3') was cloned into the lentiviral vector with mU6-MCS-Ubi-EGFP element (Shanghai Genechem, Co., Ltd., China), abbreviated to LV-sh-CAL and LV-sh-NC, respectively. The lentivirus titer units were $5 \times 10^{8} \mathrm{TU} / \mathrm{ml}$. Cells were infected with multiplicity of infection (MOI) 100 after the addition of $5 \mu \mathrm{g} / \mathrm{ml}$ polybrene, and were then treated with rotenone followed by further analysis.

Adeno-associated virus (AAV) was used in animals. For overexpression of CAL, the full length of human gene $C A L$ mRNA was cloned into the AAV9-CMV-betaGlobin-MCSEGFP-3Flag-SV40 Poly A viral vector with $K p n I$ and BamHI restriction enzymes using the Viral Vector Package service from Shanghai GeneChem. The resulting packaged virus was verified by qPCR and the AAV9-CMVbetaGlobin-hCAL-MCS-EGFP-3Flag-SV40 Poly A (abbreviated to AAV-CAL) genome titer was $1.91 \times 10^{12} \mathrm{v} . \mathrm{g} /$ $\mathrm{ml}$ and the titer of the control viral AAV9-CMV-betaGlobinMCS-EGFP-3Flag-SV40 Poly A (abbreviated to AAV-GFP) was $1.23 \times 10^{13} \mathrm{v} \cdot \mathrm{g} / \mathrm{ml}$. For knockdown of CAL, the plasmid composed of 4 connected bis-cistronic expressions of shRNA towards the sequences of rat $C A L$ mRNA was constructed, which was combined with GFP. The expression of shRNA or GFP was driven by the U6 promoter and by the CMV promoter, respectively. The cassette was flanked by pAV inverted terminal repeats (ITRs), constructed by Viagene Biosciences. The 4 corresponding shRNAs were as follows: 1,5'-GGATCTGGAAAGAGAACTT-3'; 2,5'-GGGT CCAACAAATACAGTT-3'; 3,5'-GGAAGATCATGAAG GCCTT-3'; 4,5'-GGTAATTCTGGTGCTAGTT-3'. To generate the AAV, pAV-4in1GOPCshRNA-GFP or pAV4in1shRNA-GFP was sub-cloned into the AAV9 vectors, and the viruses were purified and determined by qPCR. The genome titer of the AAV-4in1shRNA-GFP vector (abbreviated to AAV-scramble) was $2.55 \times 10^{13} \mathrm{v} . \mathrm{g} / \mathrm{ml}$ and was $2.71 \times 10^{13} \mathrm{v} \cdot \mathrm{g} / \mathrm{ml}$ in AAV-4in1GOPCshRNA-GFP (abbreviated to $\mathrm{AAV}$-shCAL).

\section{Coimmunoprecipitation and Western Blot Analysis}

The experimental procedures were described previously [32]. Briefly, cells were harvested and lysed in ice-cold lysis buffer
(10 mM HEPES, $50 \mathrm{mM} \mathrm{NaCl}, 5 \mathrm{mM}$ EDTA, $1 \mathrm{mM}$ benzamidine, $0.5 \%$ Triton $\mathrm{X}-100, \mathrm{pH} 7.4$ ) containing $1 \times$ protease inhibitor cocktail (Thermo Fisher Scientific, Rockford, IL, USA). The supernatant was first incubated with $50 \mu \mathrm{l}$ of protein $\mathrm{A} / \mathrm{G}$-agarose overnight at $4{ }^{\circ} \mathrm{C}$ followed by binding with anti-mGluR5 or anti-CAL polyclonal antibody for 4-6 h at $4{ }^{\circ} \mathrm{C}$. The precipitated complex was washed 3 times followed by elution, and then Western blot analysis was performed.

Cells or tissues were homogenized with RIPA lysis buffer (Solarbio, Beijing, China) containing $1 \mathrm{mM}$ PMSF using a rotor-stator homogenizer (Bandelin, Germany). The lysates were clarified via centrifugation at $15000 \mathrm{~g}$ for $5 \mathrm{~min}$ at $4{ }^{\circ} \mathrm{C}$. The supernatant was separated by SDS-PAGE ( 8 or $10 \%$ ) and transferred onto polyvinylidene difluoride (PVDF) membranes (Millipore Corporation, Billerica, MA, USA). The membranes were then blocked for $1 \mathrm{~h}$ with TBST $(20 \mathrm{mM}$ Tris- $\mathrm{HCl}, \mathrm{pH}$ 7.6, $137 \mathrm{mM} \mathrm{NaCl}, 0.05 \%$ Tween 20) containing 5\% skimmed milk at room temperature, and incubated with the primary antibodies at $4{ }^{\circ} \mathrm{C}$ overnight. The blots were rinsed with TBST and incubated with the appropriate horseradish peroxidase (HRP)-conjugated secondary antibody for $1 \mathrm{~h}$ at room temperature and then visualized using enhanced chemiluminescence reagents (Millipore). The primary antibodies used were as follows: monoclonal anti-TH (1:5000, Sigma-Aldrich, T1299), polyclonal antimGluR5 (1:1000, Abcam, Cambridge, MA, USA, ab53090), polyclonal anti-CAL (1:1000, Abcam, ab37036), polyclonal anti-mGluR1 (1:1000, Millipore, 07-617), and polyclonal anti-p-ERK1/2 (1:2000, Millipore, 05-797R), polyclonal anti-p-AKT (1:1000, Cell Signaling Technology, Danvers, MA, USA, 9721s), anti-p-JNK (1:1000, Cell Signaling Technology, 4668s), polyclonal anti-PARP (1:1000, Cell Signaling Technology, 9542s), polyclonal anti-caspase-3 (1:1000, Cell Signaling Technology, 9662s), and polyclonal antiubiquitin (1:1000, Cell Signaling Technology, 3933s). The intensity of the blots was quantified by Image J software (National Institutes of Health, Bethesda, MD, USA) and analyzed using GraphPad Prism 5.0 software (GraphPad Inc., La Jolla, CA, USA).

\section{Glutamate Release Assay}

MN9D cells were plated in 96-well plates at $5 \times 10^{3}$ cells/ well. After $24 \mathrm{~h}$, the cells were transfected with either the CMV-IRES-hCAL-GFP/CMV-IRES-GFP or shGFP/ shCAL plasmid, and cells transfected with the shGFP/ shCAL plasmid were pretreated with MPEP for $30 \mathrm{~min}$. Then all cells were treated with rotenone for $24 \mathrm{~h}$. The cell supernatant was obtained and the glutamate assay with the Amplex Red Glutamic Acid Assay Kit (Invitrogen, Grand Island, NY, USA) was performed. 
The reactions were incubated for $30 \mathrm{~min}$, protected from light at $37{ }^{\circ} \mathrm{C}$, and the fluorescence assayed at an excitation wavelength of $571 \mathrm{~nm}$ and emission wavelength of $590 \mathrm{~nm}$ using a microplate reader. The concentration of glutamic acid was quantified with the L-glutamate standard curve.

\section{Animals and Treatment}

Male Sprague-Dawley rats weighing $220-250 \mathrm{~g}$ at the beginning of the experiment were acclimated for 1 week. The animals were housed under a 12-h light/dark cycle at room temperature $\left(22 \pm 2{ }^{\circ} \mathrm{C}\right)$ with ad libitum access to food and water. All procedures were performed following the National Institutes of Health Guide for the Care and Use of Laboratory Animals and were approved by the Committee on Animal Care and Usage (Capital Medical University). The animals were divided into 2 groups: the control group that received vehicle injection, and the PD group that received an infusion of rotenone. For model establishment, the rats were deeply anesthetized by intraperitoneal injection of chloral hydrate $(300 \mathrm{mg} / \mathrm{kg}$; Guang Fu Fine Chemical Research Institute, Tianjin, China) and fixed in a stereotaxic frame. Rotenone was dissolved in the vehicle PEG/DMSO ( $v: v=1: 1)$ at the dose of $6 \mu \mathrm{g} / \mu \mathrm{l}$. One microliter of rotenone or vehicle was infused using a 10- $\mu$ l Hamilton syringe into the right substantia nigra $(\mathrm{SN})$ at the following stereotaxic coordinates with respect to bregma: $\mathrm{AP}=-5.0 ; \mathrm{ML}=-2.0 ; \mathrm{DV}=-7.8$. The injection speed was $0.2 \mu \mathrm{l} / \mathrm{min}$ and the needle remained for an additional $5 \mathrm{~min}$ before withdrawal. Following 2 weeks recovery, $3 \mu \mathrm{l}\left(\sim 10^{10}\right.$ total particles $)$ virus vectors was injected stereotaxically into the same coordinates through the hole in the skull. The microinjections were carried out at a rate of $0.5 \mu 1 / \mathrm{min}$ and the syringe was retained for 5 min before retraction. Either AAV-GFP and AAV-CAL viral vectors or $\mathrm{AAV}$-scramble and $\mathrm{AAV}$-shCAL were both included in the control and rotenone groups (10 animals/each group). In the rotenone group with AAVscramble or AAV-shCAL injection, animals received MPEP $(1.5 \mathrm{mg} / \mathrm{kg} /$ day $)$ by intraperitoneal injection at the same day of the virus injection for continuous 3 weeks ( 8 animals/each group). The death rate after surgery was < $10 \%$ and 8 or 9 animals remained in each group. After 4 weeks of virus delivery, the animals were killed and brain tissues obtained for further pathophysiological analysis. The sample sizes used in this study were based on estimations from a power analysis.

\section{Behavioral Assessment}

Apomorphine-induced rotation was measured 4 weeks after virus injection. Rats were injected subcutaneously with
$0.5 \mathrm{mg} / \mathrm{kg} \mathrm{R}$-(-)-apomorphine hydrochloride hemihydrate (Sigma-Aldrich) dissolved in saline containing $0.01 \%$ ascorbic acid and placed in a 30-cm-diameter bowl attached to a rotameter (Columbus Instruments, Columbus, OH, USA). The number of contralateral full body turns within 30 min was recorded. During this process, the body weight of each animal was assessed every week after the rotenone-induced lesion.

\section{Immunohistochemistry and Immunofluorescence}

After the final behavioral tests, the animals were deeply anesthetized with chloral hydrate and transcardially perfused with saline followed by ice-cold 4\% PFA (SigmaAldrich) in $0.1 \mathrm{M}$ PBS ( $\mathrm{pH} 7.4$ ). The brains were dissected and post-fixed in 4\% PFA overnight and then dehydrated with gradient sucrose solutions from 20 to $30 \%$ and then embedded in OCT compound at $-80{ }^{\circ} \mathrm{C}$. Frozen brain was coronally sectioned through the striatum and $\mathrm{SN}$ at $40-\mu \mathrm{m}$ thickness using a freezing microtome (Leica, Solms, Germany). For the immunohistochemistry assay, SN and striatum sections were used and endogenous peroxidase activity was quenched with $3 \%$ hydrogen peroxide for $10 \mathrm{~min}$ and then washed with PBS, permeabilized with PBS containing 10\% normal horse serum and $0.3 \%$ Triton $\mathrm{X}-100$, followed by incubation in blocking buffer (5\% BSA in PBS) for $1 \mathrm{~h}$ at room temperature. The sections were incubated overnight at $4{ }^{\circ} \mathrm{C}$ with the primary antibody anti-TH $(1: 2500$, SigmaAldrich, T1299), anti-mGluR5 (1:50, Abcam, ab53090), or anti-CAL (1:100, Santa Cruz, sc-393026). After 3 washes with PBS, the sections were then incubated with biotinylated anti-mouse or anti-rabbit secondary antibody (Vector Stain ABC kit, Burlingame, CA, USA) followed by staining with 3,3'-diaminobenzidine solution (DAB, Zhongshan Goldenbridge Biotechnology, Beijing, China) to visualize immunoreactivity. The sections were then mounted on cation anti-off slides (Zhongshan Goldenbridge Biotechnology), dehydrated through ascending graded concentrations of alcohol, cleared in xylene, and mounted under coverslips. Sections were imaged on a Leica Qwin microscope, and the intensity of $\mathrm{TH}^{+}$neurons or $\mathrm{TH}$ immunoreactive (TH-IR) fibers in the striatum was quantified using Image $\mathrm{J}$ software analysis. For immunofluorescence, SN sections were incubated with mouse monoclonal anti-TH (1:2500, Sigma-Aldrich, T1299) for TH/GFP dual staining, and rabbit polyclonal anti-mGluR5 (1:200, Millipore, AB5675) and mouse monoclonal anti-CAL (1:50, Santa Cruz, sc-393,026) for mGluR5/CAL dual staining overnight at $4{ }^{\circ} \mathrm{C}$. After 3 washes with PBS, the sections were then incubated with Alexa Fluor 594 donkey anti-mouse (1:200, Invitrogen, A-21203), or Alexa Fluor 594 donkey anti-rabbit (1:200, Invitrogen, A-21207) and Alexa Fluor 488 donkey anti- 
mouse (1:200, Invitrogen, A-21202) secondary antibodies for $1 \mathrm{~h}$ in the dark at the room temperature. The nuclei were then stained with 4',6-diamidino-2-phenylindole (DAPI, Cell Signaling Technology) for $5 \mathrm{~min}$ and the sections were mounted on slides with anti-fluorescence quenching agent. Images were captured using a fluorescence microscope with a Leica TCS SP5 confocal system.

\section{Statistical Analysis}

All data are expressed as mean $\pm \mathrm{SEM}$ or mean $\pm \mathrm{SD}$. Data analysis was carried out using GraphPad Prism 5.0 software and statistical differences between 2 groups were determined by the unpaired Student's $t$ test and 1-way ANOVA followed by Dunnett's test for 3 or more groups. Two-way repeated ANOVA was used to determine the statistical changes in each measure. Differences were considered statistically significant at $p<0.05$.

\section{Results}

\section{CAL Modulates Group I mGluRs-Mediated ERK1/2 or AKT Signaling in C6 Astroglial Cells}

Previous studies have shown that CAL interacts with group I mGluRs to regulate their activity or expression in COS7 cells [31, 32]. To investigate the biological effect of the interaction between CAL and group I mGluRs, we first examined the association between these 2 proteins using coimmunoprecipitation in C6 astroglial cells. In agreement with previously published results, mGluR 1 was observed in the immunoprecipitated complex with anti-CAL antibody and the interaction was enhanced by stimulation with the group I mGluRs agonist DHPG (Fig. 1A, left). In addition, mGluR5 immunoprecipitation followed by Western blot analysis of CAL showed robust coimmunoprecipitation of CAL with mGluR5 and stimulation by the receptor agonist CHPG strengthened the interaction (Fig. 1A, right). We then examined the downstream AKT and ERK1/2 signaling of group I mGluRs under the conditions of CAL deficiency. To knock down the protein level, the plasmid of CAL shRNA or scramble control was transfected into $\mathrm{C} 6$ astroglial cells. The protein expression of CAL knockdown is shown in Fig. 1B. In the control shRNA group, DHPG markedly elicited $\mathrm{p}$ AKT and p-ERK1/2, while CAL knockdown blocked activation of the receptor (Fig. 1C), indicating an incremental effect of CAL on the agonist-induced activation of group I mGluRs signaling. In order to further define the manipulation of CAL on group I mGluRs activity, we used AIDA, an antagonist of the group I mGluRs, to block the receptor activity. CAL shRNA obstructed
DHPG-induced AKT and ERK1/2 phosphorylation compared to control shRNA, and this effect disappeared following administration of AIDA (Fig. 1D). Collectively, these findings show that CAL increases the downstream signaling of group I mGluRs upon DHPG activation.

DHPG activates both mGluR1 and mGluR5 [33]. To further determine the modulation of CAL on both subtype receptors, we used the antagonist MPEP to inhibit mGluR5 and LY367385 to block mGluR1, and measured the level of pAKT and p-ERK1/2 under the condition of CAL deficiency. Knockdown of CAL inhibited DHPG-induced phosphorylation of AKT or ERK1/2 when either of each antagonist was used, indicating that $\mathrm{CAL}$ regulates the activities of both mGluR1 and mGluR5 (Fig. 1E and F).

\section{CAL Enhances the Protective Effect of Group I mGluRs Against STS-Induced Cell Apoptosis in C6 Astroglial Cells}

We further assessed the physiological function of CAL. As both AKT and ERK1/2 signaling elicited by DHPG favor cell survival

Fig. 1 CAL modulates group I mGluRs-mediated AKT and ERK1/2 signaling in C6 astroglial cells. (A) Cells were treated with DHPG for $30 \mathrm{~min}$ followed by coimmunoprecipitation. The cell lysates were incubated with anti-CAL (left) or anti-mGluR5 (right) antibody coupled to protein $\mathrm{A} / \mathrm{G}$ beads to immunoprecipitate, and the complexes were detected with anti-mGluR1 (left) or anti-CAL (right) antibody via Western blotting to test the interaction (up). Total lysates were probed with antimGluR1 or anti-mGluR5 antibody to visualize the equal expression (down). (B) Cells were transiently transfected with plasmids either for CAL knockdown or scramble control. At $48 \mathrm{~h}$ after transfection, cells were harvested to detect the level of CAL using Western blotting. The protein level was normalized to $\beta$-actin and expressed as the fold difference of scramble. (C) At $48 \mathrm{~h}$ after transfection, cells were stimulated with DHPG $(100 \mu \mathrm{M}, 5 \mathrm{~min})$ followed by analysis of the activities of AKT and ERK1/2 by Western blotting (left). Protein levels were normalized to $\beta$ actin and represented as the percentage difference of the control shRNA group with DHPG stimulation in AKT (middle) or ERK1/2 (right) phosphorylation. (D) Cells after transfection were pretreated with AIDA (100 $\mu \mathrm{M}, 25 \mathrm{~min})$ followed by DHPG stimulation $(100 \mu \mathrm{M}, 5 \mathrm{~min})$, and cell lysates were detected for the activities of AKT and ERK1/2 by Western blotting (left). Protein levels were normalized to $\beta$-actin and represented as the percentage difference of the AKT (middle) or ERK1/ 2 (right) phosphorylation compared to control shRNA with DHPG stimulation. (E, F) After transfection, cells were pre-incubated with MPEP $(10 \mu \mathrm{M}, 25 \mathrm{~min})$ or LY367385 (100 $\mu \mathrm{M}, 25 \mathrm{~min})$ to block mGluR5 and mGluR1, respectively, followed by DHPG $(100 \mu \mathrm{M}, 5 \mathrm{~min})$ treatment. Samples were analyzed for the activities of AKT and ERK1/2 via Western blotting (left). Protein levels were normalized to $\beta$-actin and represented as the percentage difference of p-AKT (middle) or p-ERK1/2 (right) compared with the control shRNA. Either the control shRNA or CAL shRNA group (in panels for quantification) was calculated as the fold difference relative to its corresponding phospho-AKT or phosphoERK1/2 immunoreactivity by the presence of stimulation over the absence of stimulation. Data shown in this figure represent the means \pm SEM of 3 independent experiments. The statistical significance was determined using Student's $t$ test. * $p<0.05$ versus control shRNA group 


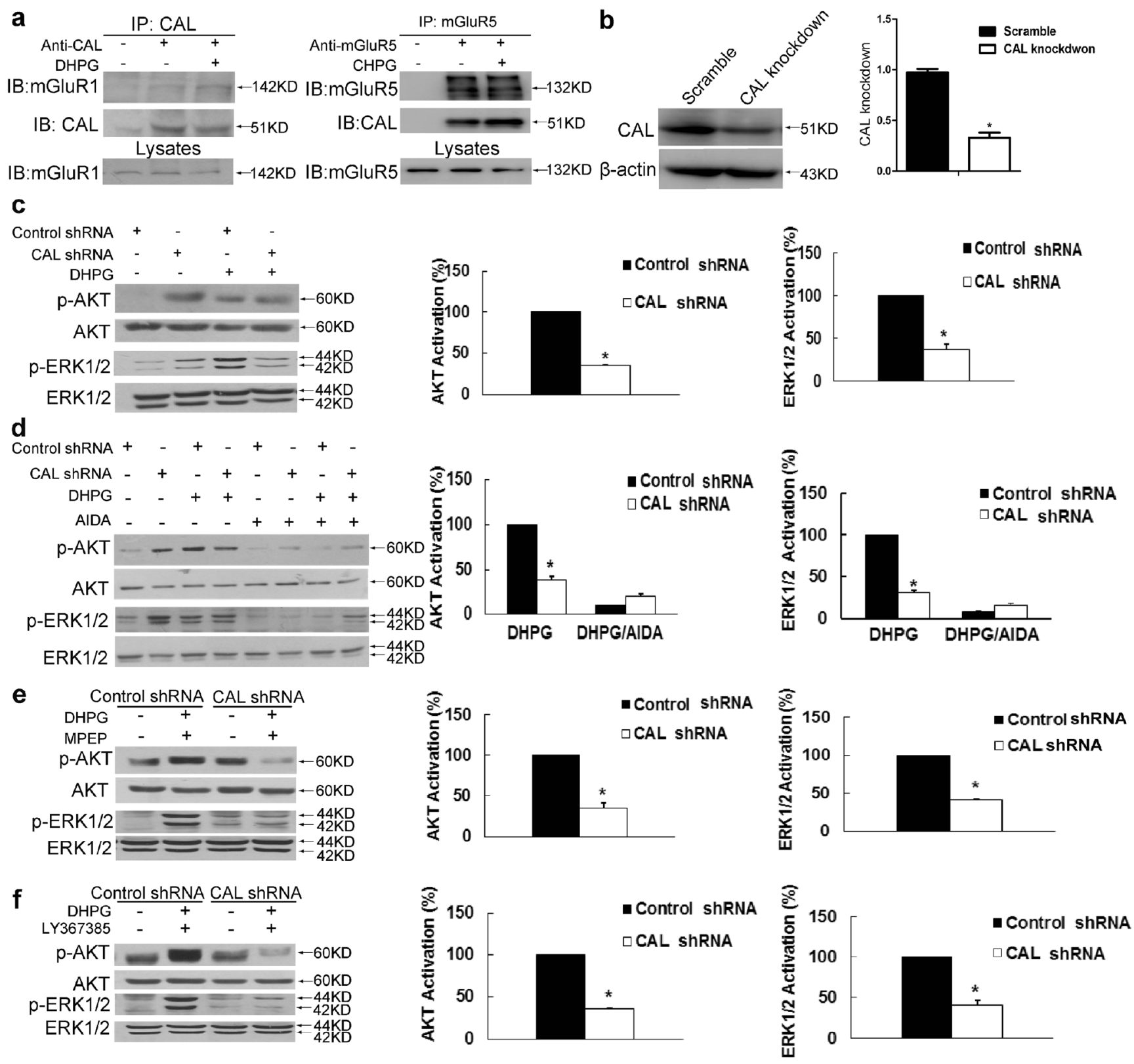

$[34,35]$, we investigated whether CAL modulated the effect of DHPG on C6 astroglial cell apoptosis. The results from Annexin V-PI and TUNEL staining assays showed that DHPG protected against STS-induced cell apoptosis, which was almost abolished when CAL was interfered (Fig. 2A and B). Consistently, the cleavage of caspase-3 and PARP, markers of cell apoptosis, was decreased in the presence of DHPG pretreatment, which was almost abolished by the knockdown of CAL (Fig. 2C). Furthermore, when AIDA was added to the cells, the protective effect of both DHPG and anti-DHPG action of CAL knockdown were abrogated, as demonstrated by the persistent high level of PARP cleavage (Fig. 2D).
The above results indicate that CAL plays an augmented role in cell protection by regulating the activity of group I mGluRs.

As mentioned above, CAL increased the DHPGelicited AKT and ERK1/2 signaling pathways and augmented the protective effect of DHPG against cell apoptosis in response to STS. We next examined whether the protection by CAL was via the regulation of AKT and ERK1/2 signaling. As shown in Fig. $2 \mathrm{E}$ and $\mathrm{F}$, the addition of LY294002 or U0126, inhibitors of PI3K and MEK, respectively, to inhibit AKT or ERK1/2 signaling, reduced the protective effect on DHPG treatment; however, these effects were partially blocked when CAL was knocked 
down. These results suggest that the protection of CAL against STS-induced apoptosis requires both AKT and ERK1/2 signaling.

\section{CAL Attenuates Cell Apoptosis by Regulating the Protein Level of mGluR5 in Rotenone-Treated MN9D Cells}

The above results indicated that CAL protected against cell apoptosis by regulating the activity of group I mGluRs. mGluR5 played a critical role in regulating dopaminergic neuronal death, which is important in PD pathogenesis [36]. Determining whether CAL regulated mGluR5 against dopaminergic neuronal death in PD may help to reveal the pathogenesis and provide a new strategy for the prevention and treatment of PD. Rotenone is an inhibitor of complex I in mitochondria and has been successfully used to produce a model of PD [37, 38]. MN9D cells were treated with rotenone, and the role of CAL was detected during dopaminergic neuronal injury. We first tested the viability of MN9D cells after treatment with different concentrations $(0-1 \mu \mathrm{M})$ of rotenone for $24 \mathrm{~h}$ using the 3-(4,5-dimethylthiazol-2-yl)-2, 5diphenyltetrazolium bromide (MTT) assay, and the results showed a concentration-dependent decrease in cell viability (Supplementary Fig. 1). Cell viability was reduced to $49.04 \%$ compared to the control at $0.2 \mu \mathrm{M}$ rotenone, and this concentration was used in subsequent experiments. We then overexpressed CAL using a lentiviral vector encoding human $C A L$ gene (abbreviated to LV-CAL) and a negative vector control (abbreviated to LV-NC). The efficiency of infection was measured by fluorescence microscopy and Western blotting (Supplementary Fig. 2). Rotenone decreased cell viability, which was significantly attenuated by LV-CAL (Fig. 3A). Rotenone caused consistent progressive apoptotic cell death, as shown by the cleavage of PARP which increased from 0 to $24 \mathrm{~h}$ during rotenone treatment; however, MN9D cells with overexpression of CAL were more resistant to rotenone, as shown by a marked reduction in PARP cleavage (Fig. 3B). To confirm the protective role of CAL against rotenoneinduced MN9D cell apoptosis, we performed TUNEL assay. As shown in Fig. 3C, the percentage of TUNEL-positive cells was increased after rotenone treatment, while overexpression of CAL greatly reduced the frequency of apoptotic cells. Collectively, these results reveal that $\mathrm{CAL}$ attenuates rotenone-induced apoptosis.

Next, we investigated whether CAL protected against cell apoptosis by regulating mGluR5 in rotenone-treated MN9D cells. We first determined the protein level of CAL and mGluR5 by Western blotting, respectively, in rotenonetreated MN9D cells. Following treatment with rotenone, both CAL and mGluR5 expression were decreased in a timedependent and dose-dependent manner (Fig. 3D and E). However, overexpression of CAL alleviated the rotenone- induced decrease in mGluR5 compared with the LV-NC group (Fig. 3F). In addition, we constructed a lentiviral vector with package plasmids of shRNA targeting the translation region of gene $C A L$ to suppress the expression of CAL (abbreviated to LV-sh-CAL) and the scramble control was LV-sh-NC. The efficiency of knockdown was measured by fluorescence microscopy and Western blotting (Supplementary Fig. 3). Contrary to the results obtained from the overexpression system, CAL knockdown aggravated mGluR5 reduction under rotenone treatment (Fig. 3G). We did not detect an alteration in the protein level of mGluR5 when CAL was either overexpressed or knocked down without rotenone treatment. These results show that CAL regulates mGluR5 protein level during rotenone treatment.

\section{CAL Modulates Ubiquitin-Proteasome-Dependent Degradation of mGluR5 in Rotenone-Treated MN9D Cells}

We next investigated how CAL regulated mGluR5 expression in response to rotenone treatment. It was reported that mGluR5 was ubiquitinated at lysine residues in both the transmembrane region and carboxyl-terminal tail followed by receptor degradation [39]. We first analyzed the effect of CAL on mGluR5 degradation. Following

Fig. 2 CAL enhances the protective effect of group I mGluRs against cell apoptosis in C6 astroglial cells. (A, B) At $48 \mathrm{~h}$ after transfection with plasmids for CAL knockdown or scramble control, cells were pretreated with DHPG $(100 \mu \mathrm{M})$ for 30 min followed by STS $(1 \mu \mathrm{M})$ stimulation for $9 \mathrm{~h}$, and then AnnexinV/PI (A) and TUNEL staining (B) assays were performed to detect the apoptotic rate of cells (left). Quantitative data were represented as the fold difference of apoptosis rate over the control shRNA group with DHPG treatment (right). (C) STS was added to lysates from transfected cells following pretreatment with DHPG $(100 \mu \mathrm{M}$, $30 \mathrm{~min}$ ) and the cleavage of caspase-3 was determined by Western blotting (up). The protein level was normalized to $\beta$-actin and represented as the fold difference of the control shRNA group with DHPG stimulation (down). (D) Cells after transfection were pretreated with AIDA $(100 \mu \mathrm{M}$, $25 \mathrm{~min})$ and then DHPG stimulation $(100 \mu \mathrm{M}, 30 \mathrm{~min})$ followed by incubation with STS. Cell lysates were obtained to detect cleaved PARP using Western blotting (up) and the protein level was normalized to $\beta$-actin and represented as the fold difference of the control shRNA group following DHPG stimulation (down). (E, F) After transfection, cells were pretreated with LY294002 $(50 \mu \mathrm{M})$ or U0126 $(20 \mu \mathrm{M})$ for 25 min to block AKT or ERK1/2 signaling before DHPG stimulation $(100 \mu \mathrm{M}, 30 \mathrm{~min})$ followed by STS incubation for $9 \mathrm{~h}$, and cell lysates were analyzed by Western blotting to determine caspase- 3 cleavage. Protein levels were normalized to $\beta$-actin and represented as the fold difference of control shRNA with DHPG stimulation. Either the control shRNA or CAL shRNA group (in panels for quantification) was calculated as the fold difference relative to its corresponding apoptosis by the presence of stimulation over the absence of stimulation. Data shown in all panels of this figure represent the means \pm SEM of 3 independent experiments. The statistical significance was determined by Student's $t$ test. $* p<0.05, * * * p<0.001$ versus control shRNA group 
a
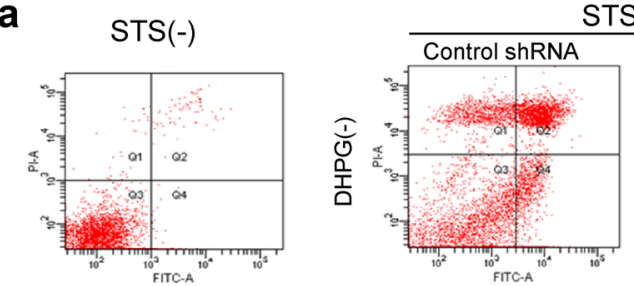

$\mathrm{TSS}(+)$
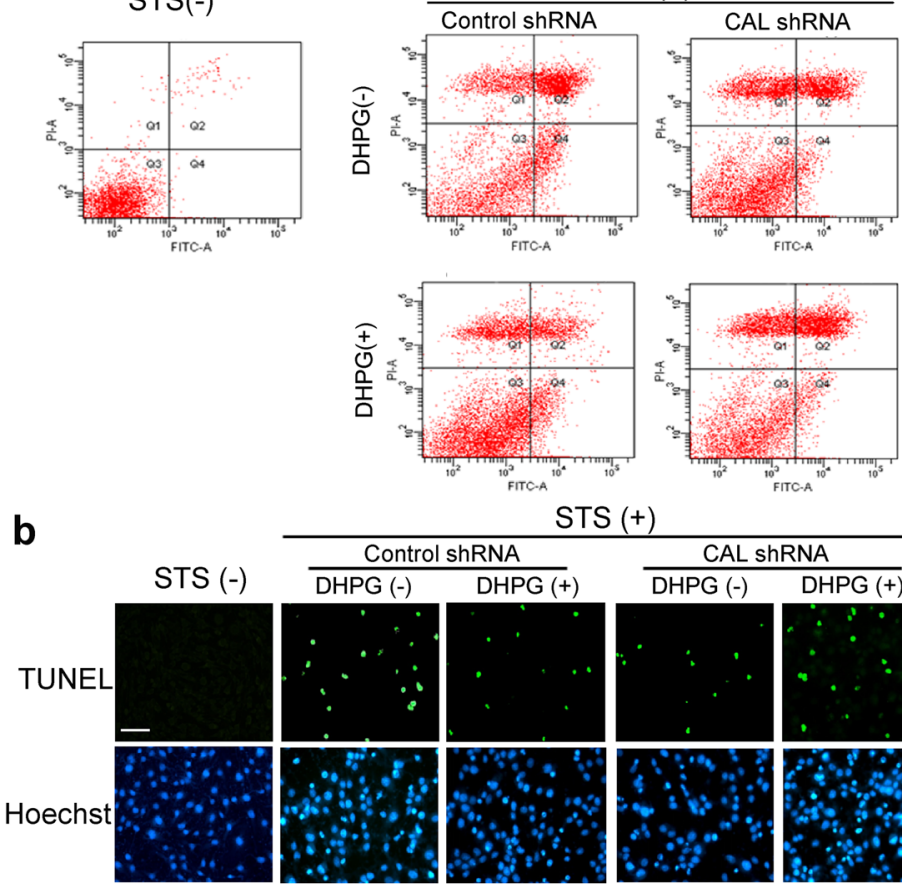

C
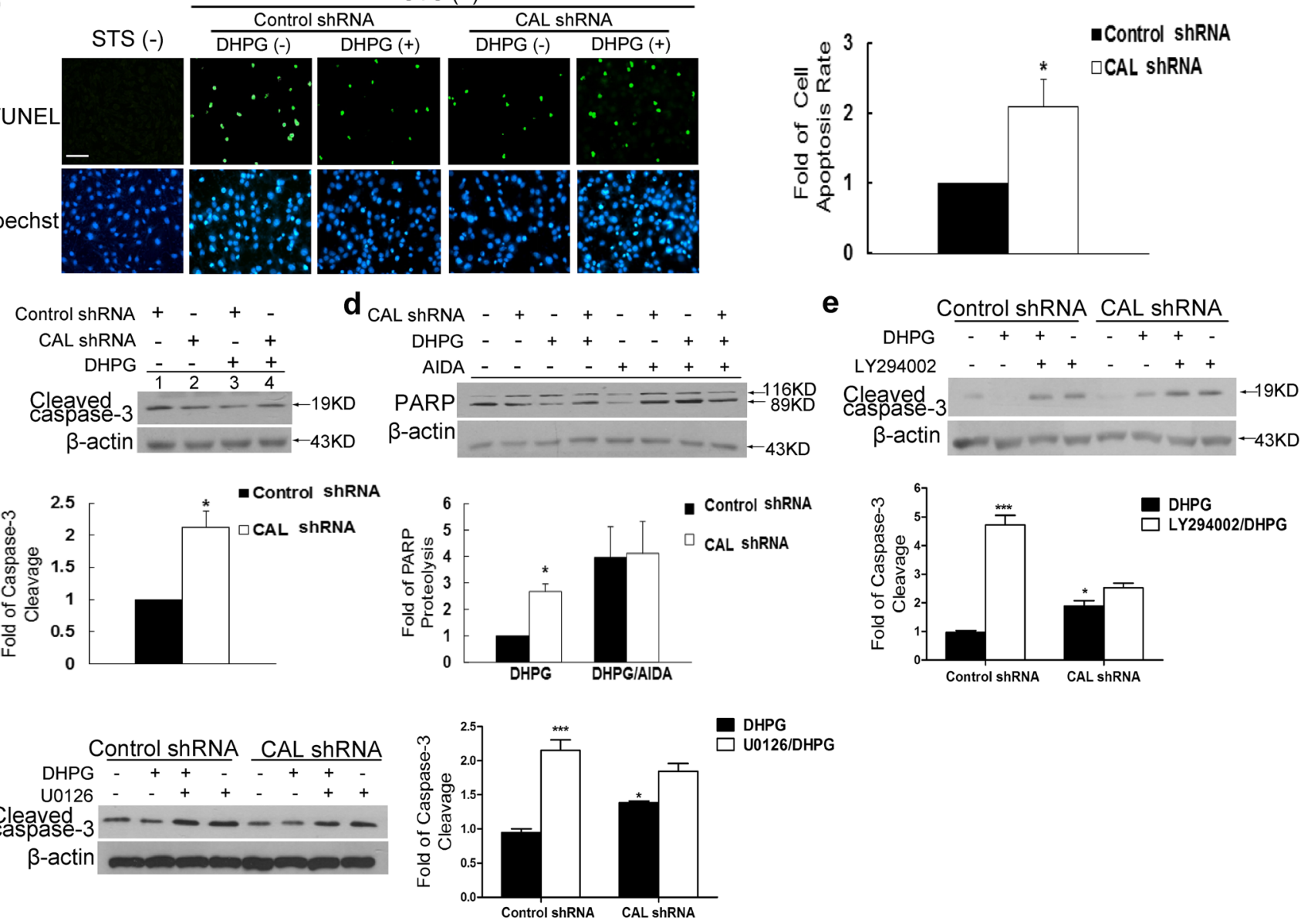

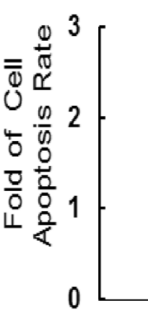

e

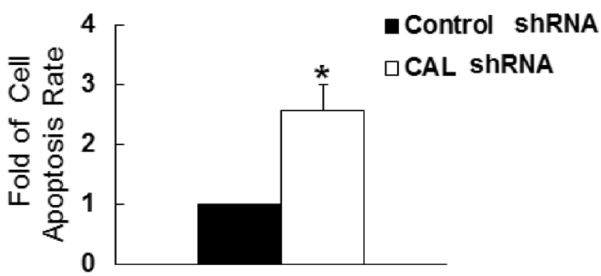

Control ShRNA

$\square$ CAL ShRNA

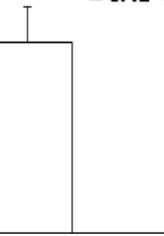

DHPG

LY294002/DHPG
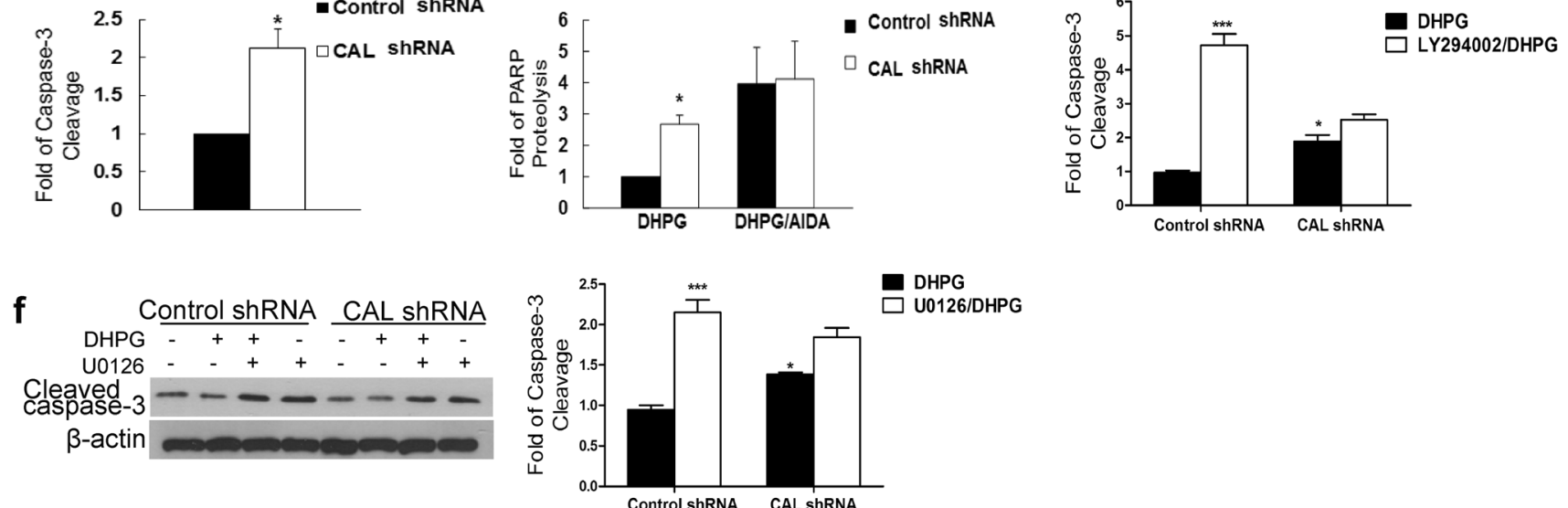

pretreatment with the protein synthesis blocker CHX followed by rotenone treatment, the remaining mGluR 5 was detected at different time points. As shown in Fig. 4A, rotenone accelerated the decline of mGluR5 protein level compared to that in the CHX-treated group. CAL overexpression decelerated the decrease in mGluR5 in response to $\mathrm{CHX}$ plus rotenone treatment compared to that in the LV-NC group (Fig. 4B), while knockdown of CAL had the opposite effect (Fig. 4C). These results indicate that CAL impedes post-translational mGluR5 degradation to increase the stability of mGluR5 in response to rotenone treatment.

To examine whether CAL regulated the ubiquitinproteasome-mediated mGluR5 degradation following rotenone exposure, we performed a ubiquitination assay after immunoprecipitation using anti-mGluR5 antibody in rotenonetreated MN9D cells. As shown in Fig. 4D, rotenone treatment markedly increased the ubiquitination of mGluR5, which was 
a

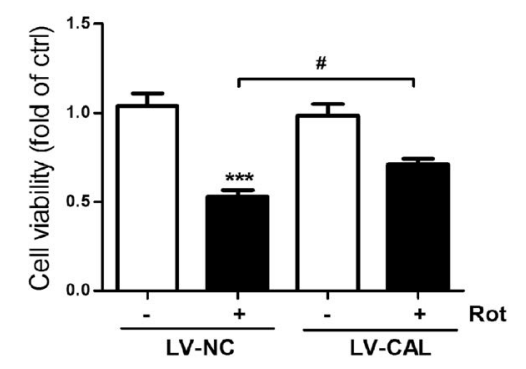

C
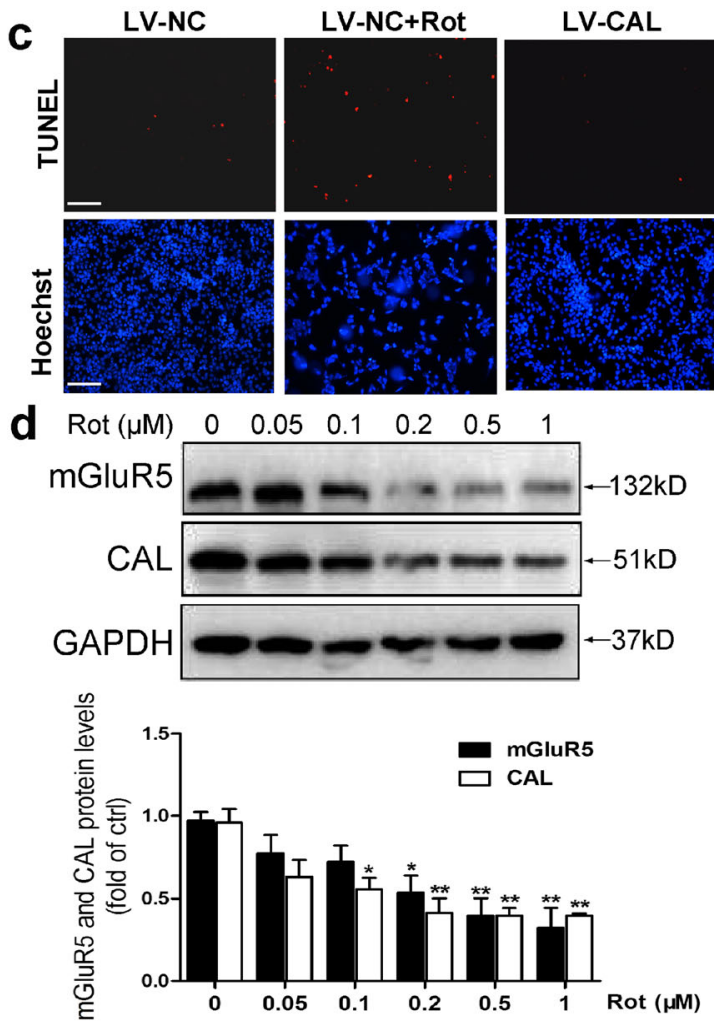

f
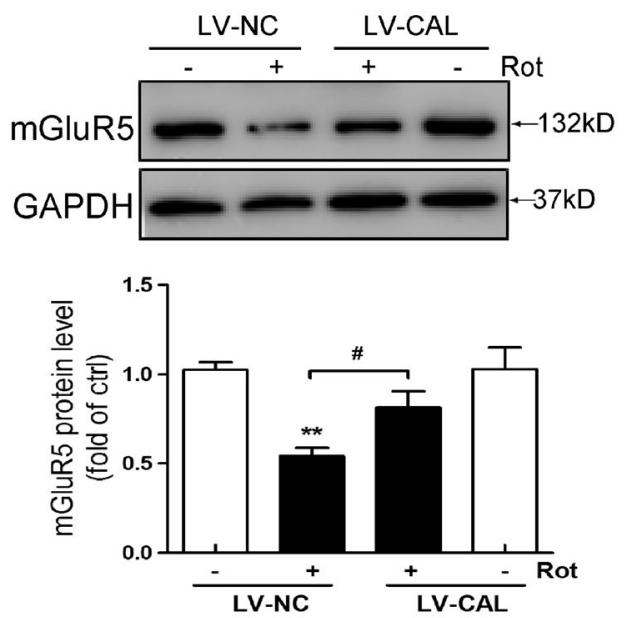

alleviated when CAL was overexpressed. Furthermore, the binding between CAL and mGluR5 was reduced when exposed to rotenone, and CAL overexpression restored the binding of CAL with mGluR5, suggesting that CAL interfered
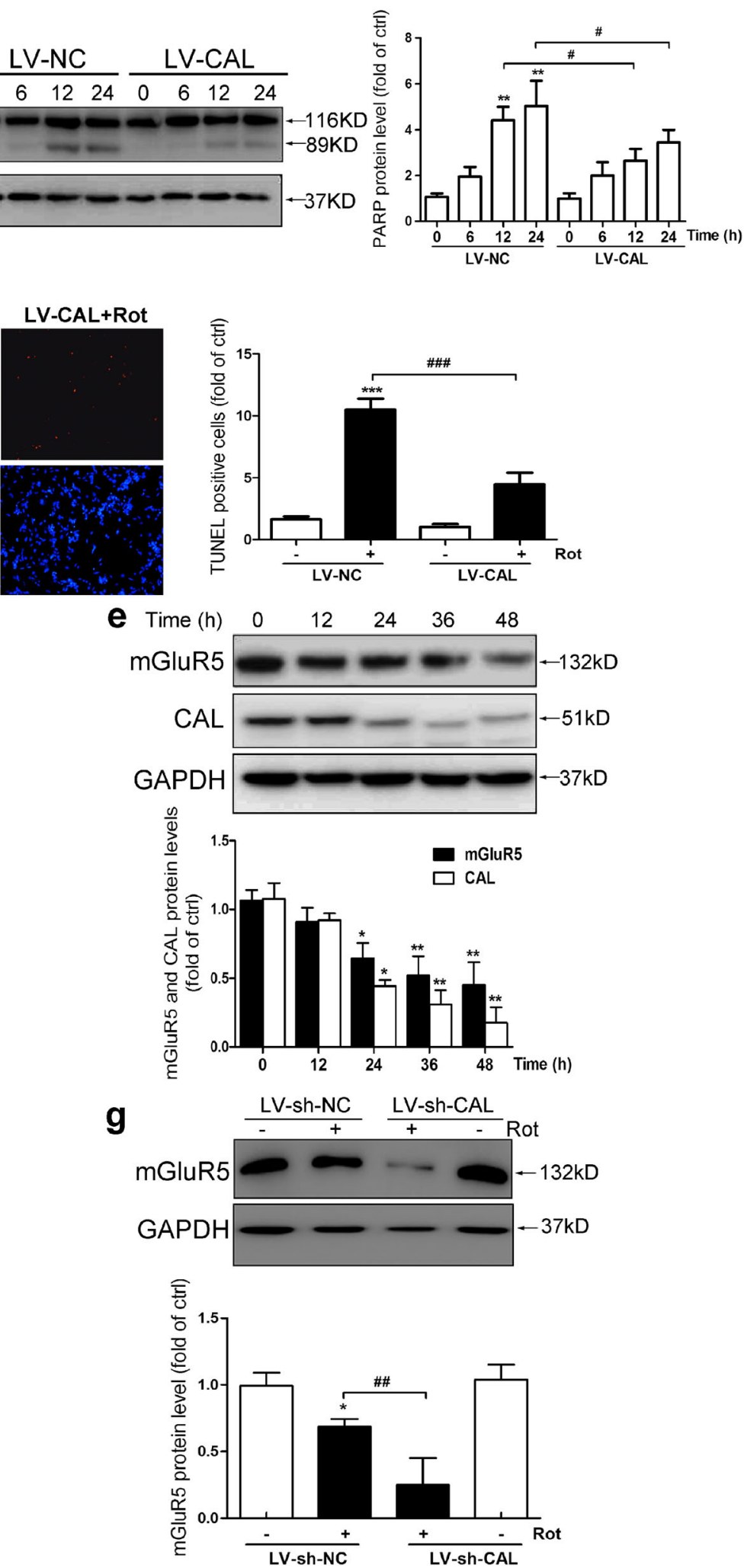

with mGluR5 ubiquitination by interacting with mGluR5 in response to rotenone treatment. In addition, the proteasome inhibitor MG132 inhibited rotenone-induced mGluR5 degradation in a dose-dependent manner (Fig. 4E). Taken together, 
Fig. 3 CAL attenuates rotenone-induced cell death by regulating mGluR5 expression in MN9D cells. (A, C) Cells were infected with LV-CAL or LV-NC followed by rotenone $(0.2 \mu \mathrm{M})$ treatment for $24 \mathrm{~h}$. The cells were then subjected to the cell viability assay (A) and cell apoptosis analysis via TUNEL staining (C). Scale bar $=100 \mu \mathrm{m}$. (B) Cells infected with LV-CAL or LV-NC were treated with $0.2 \mu \mathrm{M}$ rotenone for different time periods and then cleaved PARP was determined by Western blotting (left). The protein level was normalized to GAPDH and represented as the fold difference of the control group (right). (D, E) Cells were treated with different doses of rotenone $(0,0.05,0.1,0.2$, $0.5,1 \mu \mathrm{M})$ for $24 \mathrm{~h}(\mathrm{D})$ or $0.2 \mu \mathrm{M}$ rotenone for different times $(0,12,24$, $36,48 \mathrm{~h})(\mathrm{E})$, and cell lysates were obtained to detect mGluR5 and CAL protein levels using Western blotting (up). Protein levels were normalized to GAPDH and represented as the fold difference of the control group (down). (F) Cells were infected with LV-CAL or LV-NC followed by $0.2 \mu \mathrm{M}$ rotenone treatment for $24 \mathrm{~h}$, and lysates were obtained to detect mGluR5 expression (up). The protein level was normalized to GAPDH and represented as the fold difference of the control group (down). (G) After infection with LV-sh-CAL or LV-sh-NC, cells were exposed to rotenone $(0.2 \mu \mathrm{M})$ for $24 \mathrm{~h}$ and analyzed for mGluR5 protein level (up). The protein level was normalized to GAPDH and represented as the fold difference of the control group (down). Untreated cells were used as controls (D, E). Cells infected with LV-NC without rotenone treatment were used as controls (A-C, F). Cells infected with LV-sh-NC without rotenone treatment were used as controls $(\mathrm{G})$. Ctrl: control; Rot: Rotenone. Data shown in this figure represent the mean \pm SEM of 3 separate experiments. The statistical significance was determined by $1-$ way ANOVA followed by Dunnett's test (A, C-G). A comparison of different times of rotenone treatment between LV-CAL and LV-NC was carried out using 2-way ANOVA analysis (B). $* p<0.05$, ** $p<0.01$, and *** $p<0.001$ versus control; ${ }^{\#} p<0.05,{ }^{\# \#} p<0.01$, and ${ }^{\# \#} p<0.001$ compared to the LV-NC/LV-sh-NC group subjected to corresponding rotenone treatment

our findings reveal that CAL stabilizes mGluR5 expression by inhibiting the ubiquitin-proteasome-dependent degradation process.

\section{CAL Attenuates Rotenone-Induced Cell Apoptosis Via mGluR5-Mediated Downstream JNK Signaling in MN9D Cells}

JNK signaling transduction is believed to be activated in PD $[40,41]$. In order to determine whether JNK signaling was involved in CAL regulated cell death, we determined the level of JNK phosphorylation in MN9D cells with or without CAL overexpression after rotenone treatment. As shown in Fig. 5A, rotenone treatment increased JNK phosphorylation, which was reversed by overexpression of CAL. Correspondingly, JNK inhibition by inhibitor SP600125 alleviated the cleavage of PARP induced by rotenone (Fig. 5B). The MTT assay further showed that both SP600125 treatment and CAL overexpression attenuated the decrease in cell viability following rotenone treatment (Fig. 5C). These results suggested that CAL alleviated cell apoptosis possibly by suppressing JNK phosphorylation. To confirm the effect of CAL on JNK phosphorylation suppression by the regulation of mGluR5, MPEP was introduced into the system. MPEP predominantly suppressed JNK phosphorylation caused by rotenone, which was consistent with the results obtained when CAL was overexpressed (Fig. 5D). Taken together, these results reveal that JNK phosphorylation correlates with rotenone-induced cell damage and CAL plays a protective role by inhibiting mGluR5-mediated JNK activation.

\section{CAL Inhibits Rotenone-Induced Release of Glutamate to Modulate mGluR5 Activity}

Glutamate receptor overstimulation is indicated in excitotoxicity [42]. We investigated whether the protection by CAL against rotenone-induced toxicity was attributed to modulation of mGluR5 activity. We performed a rescue experiment. MN9D cells with CAL overexpression were exposed to rotenone followed by DHPG treatment. As shown in Fig. 6A, overexpression of CAL reversed mGluR5 degradation caused by rotenone; however, DHPG blocked the protective effect of CAL on mGluR5 protein level. Accordingly, CAL overexpression-attenuated JNK phosphorylation was reversed following stimulation by DHPG (Fig. 6B). In addition, the protein level of tyrosine hydroxylase $(\mathrm{TH})$, the ratelimiting enzyme in dopamine synthesis, was decreased accompanied by a decline in cell viability following exposure to rotenone, while CAL overexpression reversed rotenoneinduced loss of TH, which was blocked by DHPG treatment (Fig. 6C and D). These results suggest that CAL may have a beneficial effect on inhibiting excitotoxicity to appropriately modulate mGluR5 protein expression. Indeed, we found that the rotenone-induced elevation in glutamate release was inhibited by CAL expression (Fig. 6E). As previously reported, stimulation of mGluR5 facilitates glutamate release in the striatum, and MPEP exerts neuroprotection by blocking glutamate release [43]. As shown in Fig. 6F, rotenone-induced glutamate increase was blocked by the antagonist MPEP. We found that the inhibition of glutamate release by MPEP was blocked by CAL knockdown. Correspondingly, the reduced protein level of mGluR5 caused by rotenone was reversed by MPEP treatment; however, knocking down CAL blocked this effect (Fig. 6G). Collectively, these data indicate that CAL plays a role in the inhibition of excitotoxicity to modulate mGluR5 activity by regulating its protein level.

\section{CAL Overexpression Upregulates mGluR5 Expression to Play a Protective Role in the Rotenone-Induced Animal Model of PD}

As noted above, we showed that CAL had a protective function against rotenone-induced cell injury by regulating mGluR5 protein degradation and the downstream JNK signaling pathway in vitro. A rotenone-induced rat model of PD was used to corroborate these results in vivo. The AAV9 virus encoding the CAL gene (AAV-CAL) or GFP (AAV-GFP) was injected into the right substantia nigral (SN) at 2 weeks 
a

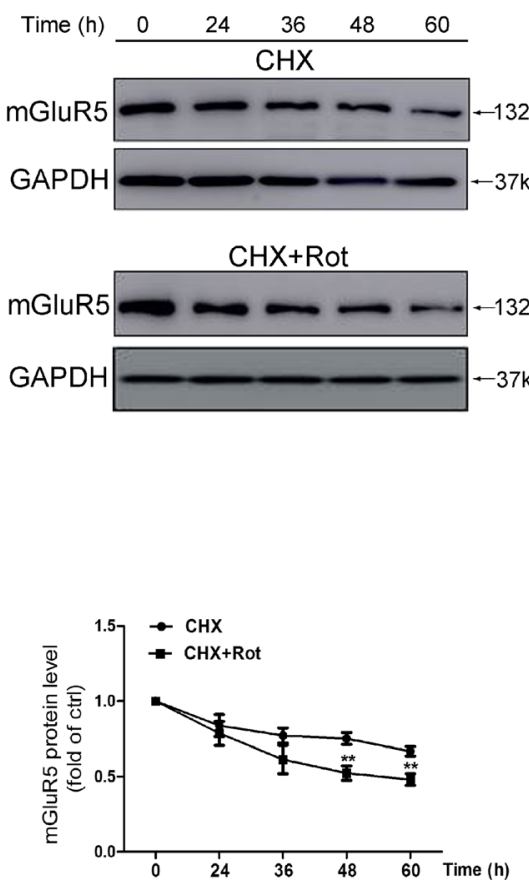

d
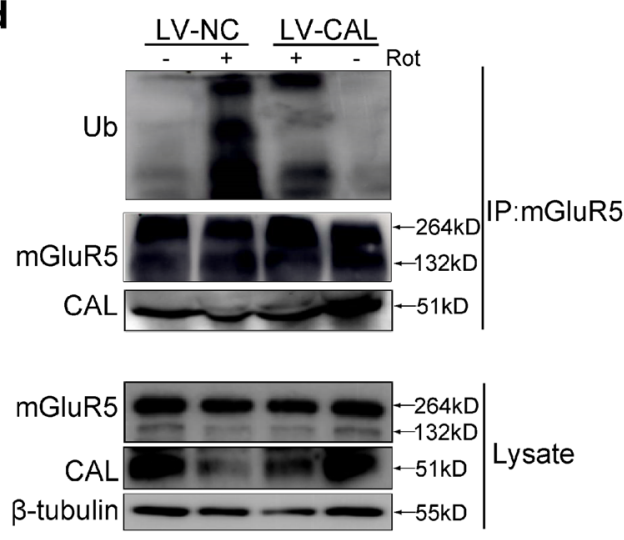

b

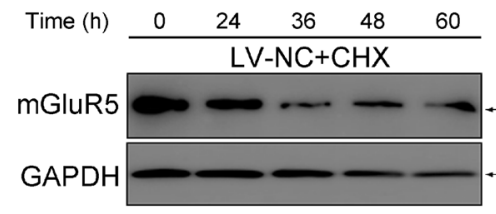

$\mathrm{LV}-\mathrm{NC}+\mathrm{CHX}+\mathrm{Rot}$

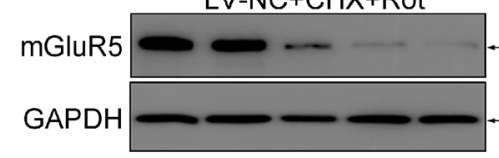

$\mathrm{LV}-\mathrm{CAL}+\mathrm{CHX}+\mathrm{Rot}$
C

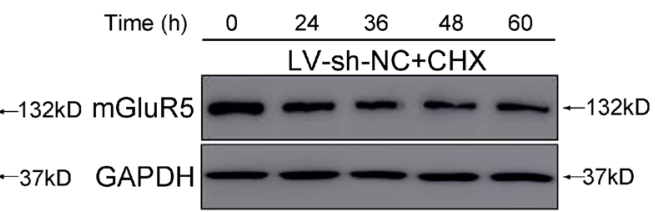

LV-sh-NC+CHX+Rot
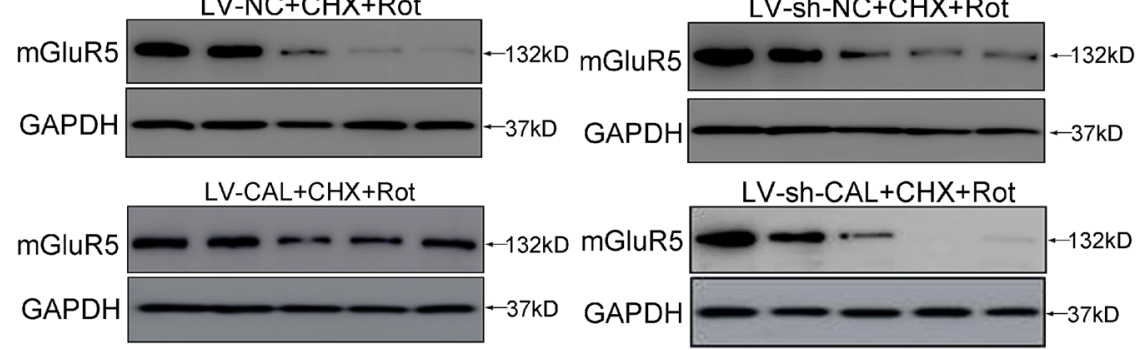

LV-sh-CAL+CHX+Rot
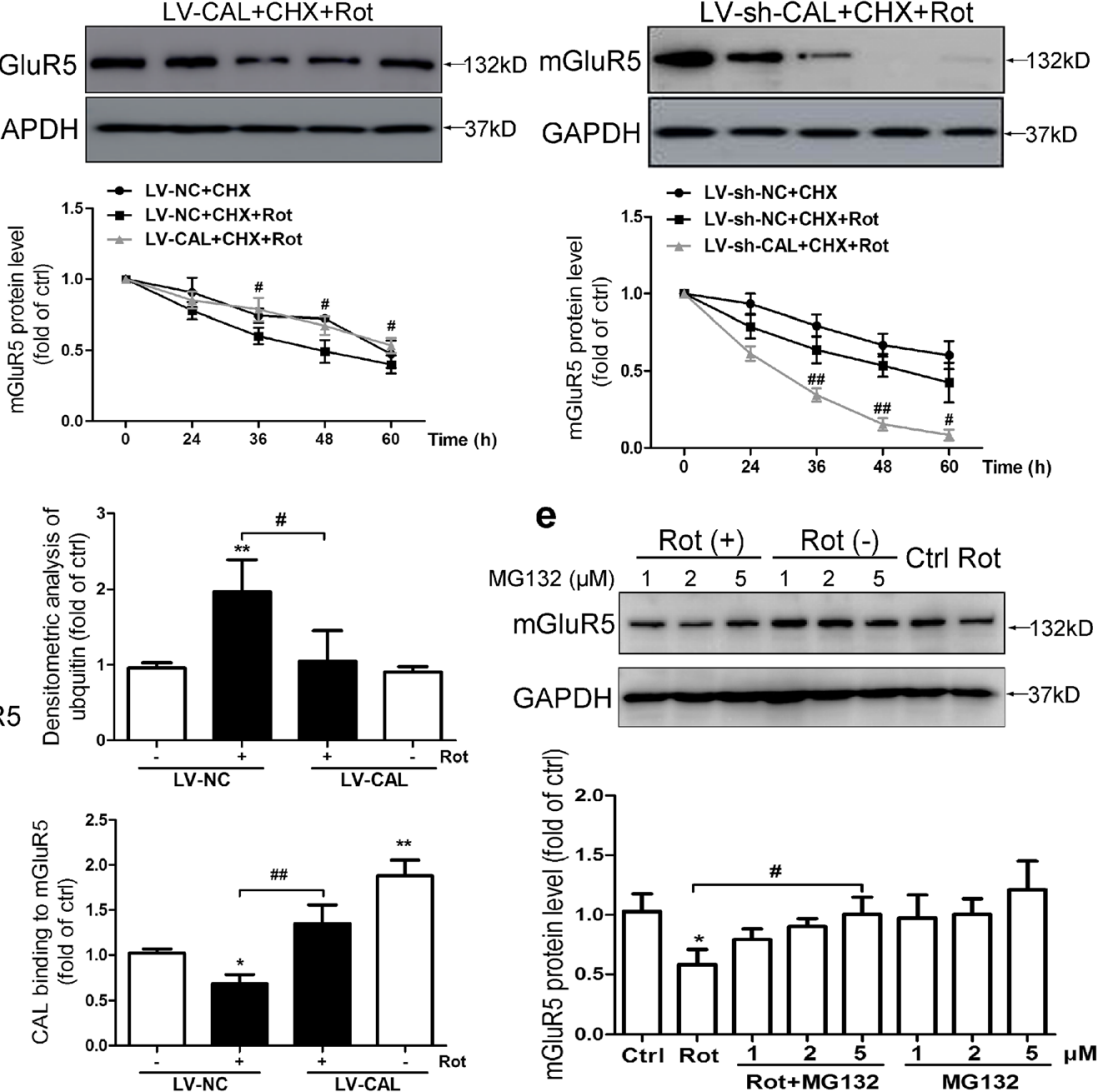

Fig. $4 \mathrm{CAL}$ inhibits ubiquitin-proteasome-dependent degradation of mGluR5 protein in rotenone-treated MN9D cells. (A) Cells were treated with $0.2 \mu \mathrm{M} \mathrm{CHX}$ for $1 \mathrm{~h}$ followed by rotenone exposure for $0-60 \mathrm{~h}$ and then Western blotting was performed to detect the alteration in mGluR5 protein level (up). The protein level was normalized to GAPDH and represented as the fold difference of the control group (down). Untreated cells were used as controls. $* * p<0.01$ versus the CHX group. (B, C) Cells with LV-CAL/LV-NC (B) or LV-sh-CAL/LV-sh-NC infection $(\mathrm{C})$ were treated with $0.2 \mu \mathrm{M} \mathrm{CHX}$ for $1 \mathrm{~h}$ followed by rotenone exposure for $0-60 \mathrm{~h}$ and then Western blotting was performed to detect the alteration in mGluR5 protein levels (up). Protein levels were normalized to GAPDH and represented as the fold difference of the control group. Cells treated with the drug for $0 \mathrm{~h}$ in the LV-CAL/LV-NC or LVsh-CAL/LV-sh-NC group were used as controls. ${ }^{\#} p<0.05,{ }^{\# \# p} p<0.01$ compared to the corresponding $\mathrm{CHX}+\mathrm{Rot}$ group. (D) Cells were infected with LV-CAL or LV-NC and treated with $0.2 \mu \mathrm{M}$ rotenone for $24 \mathrm{~h}$. The cell lysates were then subjected to immunoprecipitation with antimGluR5 antibody coupled to beads, and the precipitated complexes were probed with anti-CAL antibody to examine the interaction between CAL and the anti-ubiquitin antibody to evaluate mGluR5 ubiquitination using Western blotting. The whole lysates were probed with anti-mGluR5 and anti-CAL antibodies to visualize the expression of mGluR5 and CAL (left). Protein levels were normalized to immunoprecipitated mGluR5 and represented as the fold difference of the control group (right). Cells infected with LV-NC without rotenone treatment were used as controls. ${ }^{*} p<0.05$ and ${ }^{*} p p<0.01$ versus control group, ${ }^{\#} p<0.05$ and ${ }^{\# \# \#} p<0.01$ compared to the LV-NC+rotenone group. (E) Cells were pretreated with MG132 $(1,2,5 \mu \mathrm{M})$ for $30 \mathrm{~min}$ followed by rotenone $(0.2 \mu \mathrm{M})$ treatment for $24 \mathrm{~h}$ and mGluR5 expression was detected by Western blotting (up). The protein level was normalized to GAPDH and represented as the fold difference of the control group (down). Untreated cells were used as controls. $* p<0.05$ versus control group, ${ }^{\#} p<0.05$ compared to the rotenone group. Data shown in all panels of this figure represent the mean \pm SEM of 3 independent experiments. The statistical significance was determined by 1-way ANOVA followed by Dunnett's test (D, E) or 2-way ANOVA (A-C) 

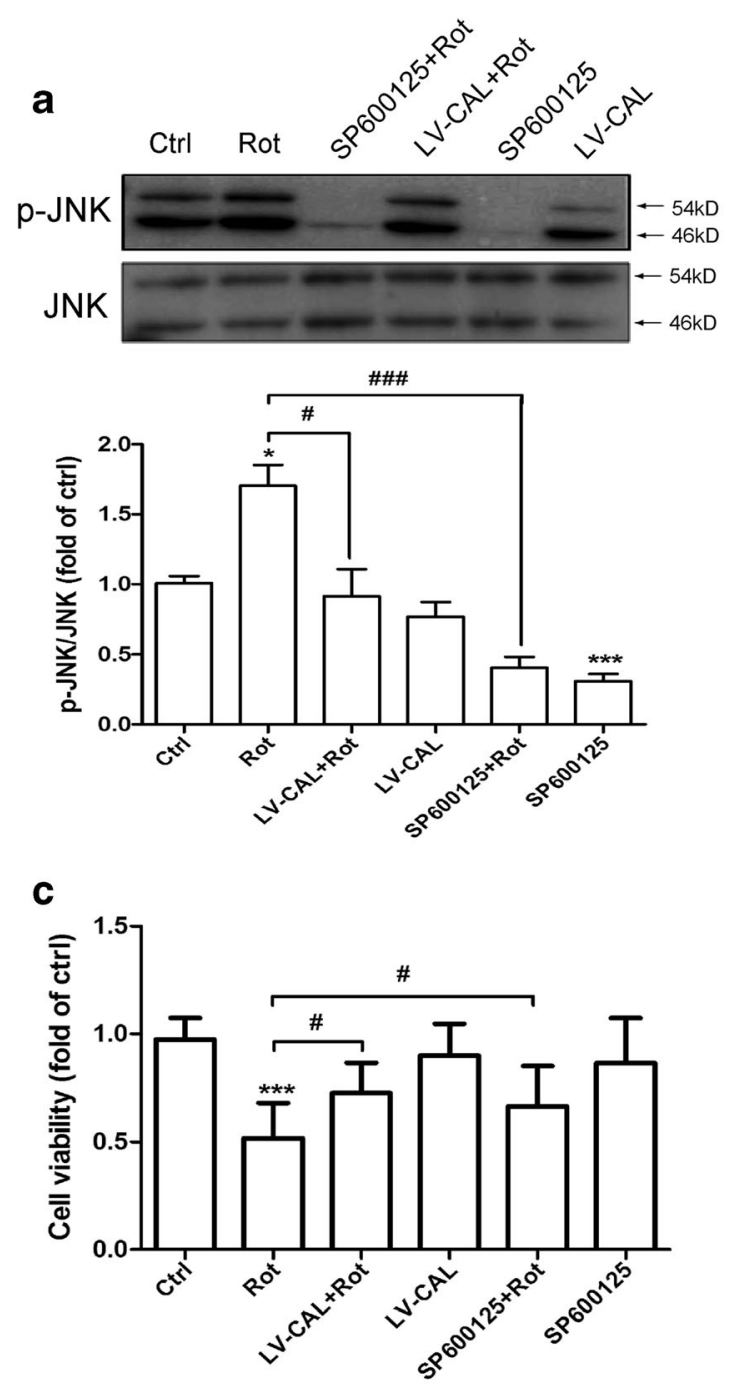

Fig. 5 CAL suppresses mGluR5-mediated JNK phosphorylation and ameliorates MN9D cell apoptosis induced by rotenone administration. (A) Cells with LV-CAL infection or SP600125 pretreatment were serum-starved for $16 \mathrm{~h}$ followed by rotenone treatment $(0.2 \mu \mathrm{M})$ for $1 \mathrm{~h}$, and then harvested for $\mathrm{p}-\mathrm{JNK}$ analysis (up). The protein level was normalized to total JNK and represented as the fold difference of the control group (down). (B) Cells were pretreated with SP600125 $(100 \mu \mathrm{M}, 30 \mathrm{~min})$ followed by rotenone $(0.2 \mu \mathrm{M})$ for $24 \mathrm{~h}$ and then harvested for PARP detection by Western blotting (up). The protein level was normalized to GAPDH and represented as the fold difference of the control group (down). (C) Cells were infected with LV-CAL or pretreated

after the rotenone lesion. Four weeks after virus injection, the behavioral performance of rats was assessed and the animals were then killed for pathophysiological analysis. The treatment scheme is shown in Fig. 7A. The right SN sections
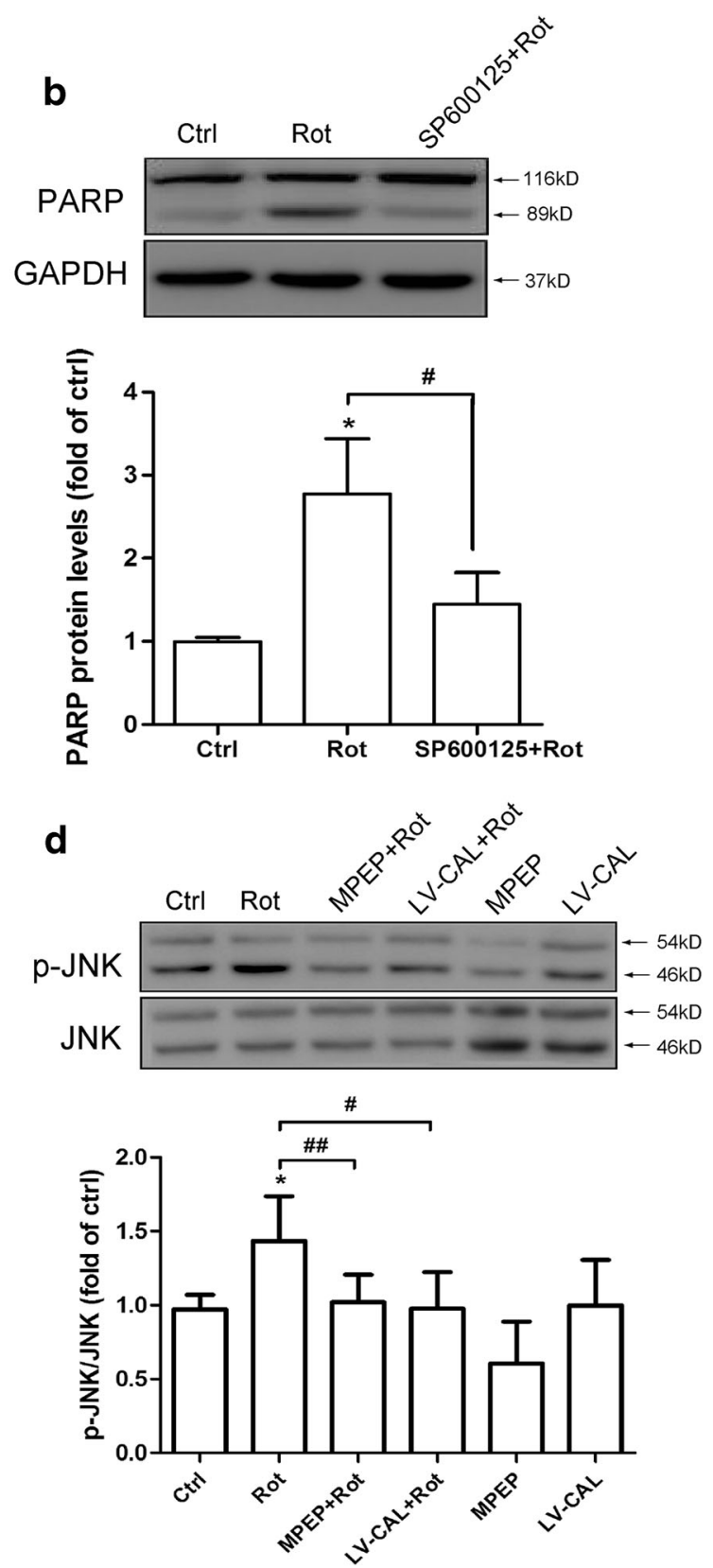

with SP600125 $(100 \mu \mathrm{M}, 30 \mathrm{~min})$ followed by rotenone $(0.2 \mu \mathrm{M})$ treatment for $24 \mathrm{~h}$, and then cell viability was estimated by the MTT assay. (D) Cells were pretreated with MPEP $(100 \mu \mathrm{M}, 30 \mathrm{~min})$ or infected with LVCAL followed by rotenone $(0.2 \mu \mathrm{M})$ for $1 \mathrm{~h}$, then cell lysates were collected for $\mathrm{p}-\mathrm{JNK}$ assessment (up). The protein level was normalized to total JNK and represented as the fold difference of the control group (down). Untreated cells were used as controls. Data shown in all panels of this figure represent the mean \pm SEM of 3 independent experiments. The statistical significance was determined by 1 -way ANOVA followed by Dunnett's test. $* p<0.05$ and $* * * p<0.001$ versus control, ${ }^{\#} p<0.05$, ${ }^{\# \#} p<0.01$, and ${ }^{\# \# \#} p<0.001$ compared to the rotenone group

displayed a homogeneous and widespread staining pattern of GFP fluorescence, whereas no GFP deposition was observed in the intact hemisphere. Double immunofluorescence staining showed intracellular localization of CAL in TH-positive 
a
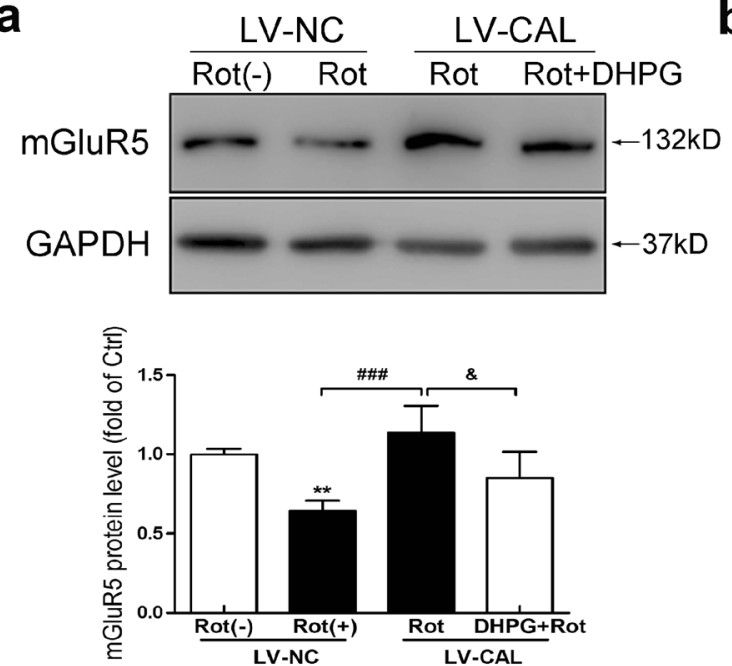

C

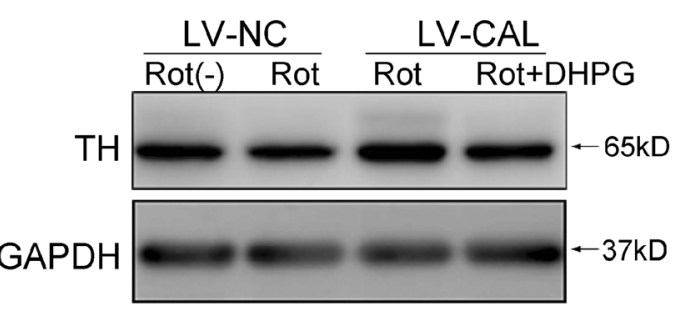

b
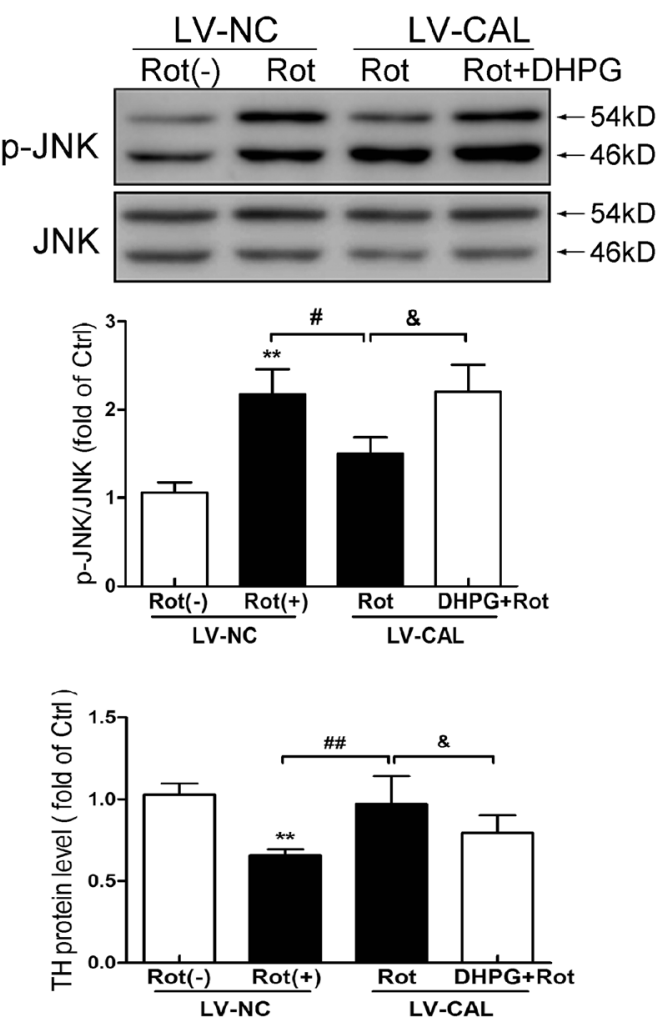

d

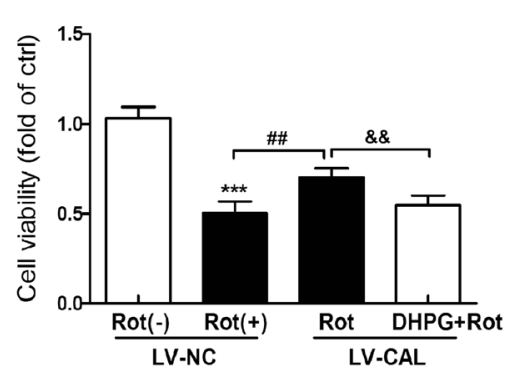

e

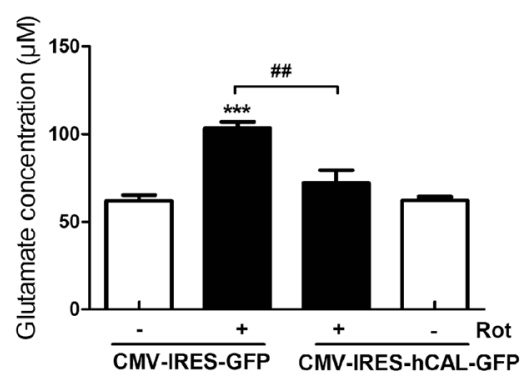

f

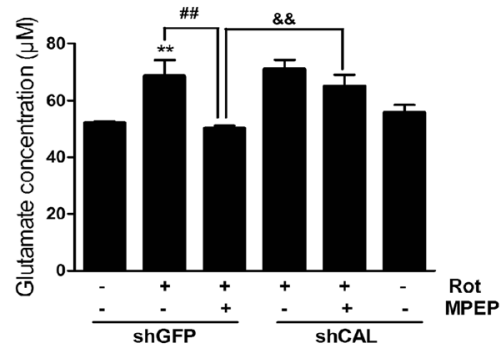

g
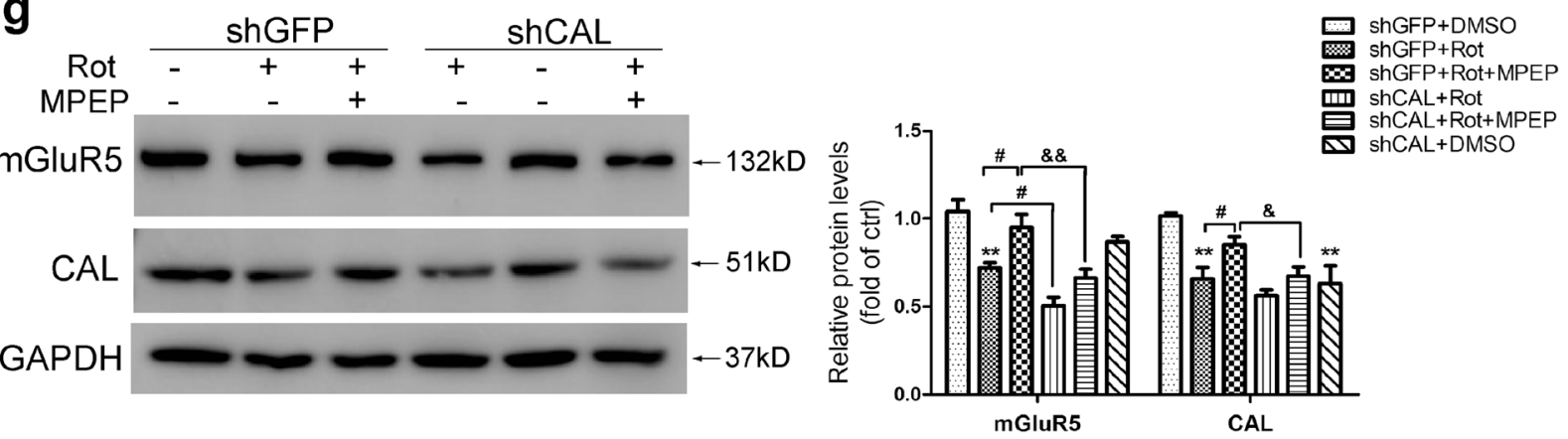

neurons. Correspondingly, brain lysates from the right side of the SN showed a higher level of CAL protein relative to the AAV-GFP control (Fig. 7B). To evaluate the beneficial role of CAL on rotenone-induced PD symptoms, apomorphineinduced rotation was carried out. All PD rats exhibited distinct apomorphine-induced rotations ipsilateral to the rotenone lesion side, while control animals (vehicle+AAV-GFP group) did not show asymmetric rotational behavior. CAL overexpression alleviated rotational behavior (Rot+AAV-CAL group). During this test, no difference in rat body weight between the vehicle and rotenone infusion groups was observed before or after virus injection (Fig. 7C). Moreover, the reduced densities of TH-positive immunoreactive cell bodies in the $\mathrm{SN}$ - and TH-positive immunoreactive fibers in the striatum 
Fig. $6 \mathrm{CAL}$ inhibits rotenone-induced glutamate release to modulate mGluR5 activity. (A-D) Cells infected with LV-CAL or LV-NC were exposed to rotenone followed by DHPG treatment, and the alteration in protein levels of mGluR5 (A), p-JNK (B), TH (C), and cell viability (D) was evaluated. Protein levels shown in corresponding graphs were normalized to GAPDH or total JNK and represented as the fold difference of the control group. (E) Cells were transfected with CMV-hCAL-IRESZsGreen or vector CMV-IRES-ZsGreen followed by $0.2 \mu \mathrm{M}$ rotenone treatment for $24 \mathrm{~h}$ and then the supernatant was assessed for glutamate concentration. (F, G) Cells transfected with shGFP or shCAL were pretreated with MPEP $(100 \mu \mathrm{M}, 30 \mathrm{~min})$ followed by rotenone for $24 \mathrm{~h}$, the supernatant was obtained for the glutamate assay $(\mathrm{F})$, and the cell lysates were probed with anti-mGluR5 and anti-CAL antibodies by Western blotting $(\mathrm{G})$. Protein levels were normalized to GAPDH and represented as the fold difference of the control group. LV-NC infected cells without rotenone treatment were used as controls (A-D). Cells transfected with vector without rotenone administration were used as controls (E-G). Data shown in all panels of this figure represent the mean \pm SEM of 3 independent experiments. The statistical significance was determined by 1-way ANOVA followed by Dunnett's test. $* * p<0.01$ and $* * * p<0.001$ versus control, ${ }^{\#} p<0.05,{ }^{\# \#} p<0.01$, and ${ }^{\# \# \#} p<0.001$ compared to the rotenone treatment group, ${ }^{\&} p<0.05$ and $\& \& p<0.01$ compared to the rotenone-treated LV-CAL group or rotenone- and MPEP-treated shGFP group

induced by rotenone administration were both alleviated by CAL administration, while no such effect was observed in the vector control AAV-GFP group (Fig. 7D).

To examine whether the protective function of CAL against rotenone-induced cell death was due to regulation of mGluR5 expression and the JNK signaling pathway in vivo, the protein levels of mGluR5 and phosphorylation of JNK in brain lysates from the striatum were examined by Western blotting. Accompanied by an alteration in $\mathrm{TH}$ expression, rotenone decreased mGluR5 and CAL protein levels and increased JNK phosphorylation, which was reversed by CAL overexpression (Fig. 7E). These results were also observed following evaluation of mGluR5 and CAL immunoreactivity in the SN (Fig. 7F). Taken together, these data indicate that CAL plays a protective role by associating with mGluR5 to modulate receptor expression and JNK signaling in rotenone-induced PD animal model.

\section{Effect of CAL Deficiency on Rotenone-Induced Toxicity In vivo}

To further corroborate the function of CAL, AAV9 encoding the shRNA sequences targeting the CAL gene (AAV-shCAL) or the scramble vector (AAV-scramble) were identically infused into the right SN 2 weeks after rotenone administration. Four weeks after virus delivery, GFP was expressed in TH-positive neurons in the right SN and no GFP deposition was observed in the intact hemisphere. This was accompanied by a lower CAL protein level due to virus interference compared with the scramble group (Fig. 8A). The assessment of apomorphine-induced rotations showed more severe behavioral abnormalities in PD rats with CAL deficiency (Rot+AAV-shCAL group) compared with the rotenone infusion (Rot+AAV-scramble) group. In addition, during virus transduction, animals had a constant body weight increase in both the vehicle and rotenone groups (Fig. 8B). Contrary to the effect of overexpression, knockdown of CAL aggravated the loss of TH-positive immunoreactive neurons and TH-positive immunoreactive fibers (Fig. 8C), accompanied by a decrease in the level of mGluR5 and an increase in the phosphorylation of JNK (Fig. 8D). These results were confirmed by measuring the immunoreactivity of mGluR5 and CAL in the SN (Fig. 8E). Taken together, these data indicate that CAL exerts a beneficial effect to mitigate symptoms in the neurotoxin rotenone-induced animal model of PD.

\section{CAL Exerts the Protective Function by Interacting mGluR5 and Inhibiting its Degradation In vivo}

As aforementioned, mGluR5 protein decreased upon the rotenone toxicity was due to the ubiquitin-mediated degradation, while overexpression of CAL stabilized mGluR5 expression via interacting with the receptor to inhibit the ubiquitinproteasome-dependent degradation process in MN9D cells, as shown in Fig. 4D. In order to corroborate this point, we detected the mGluR5-binding ubiquitin and the interaction of mGluR5 with CAL by coimmunoprecipitation assay in the rotenone-induced PD animal model. Rotenone lesion increased the ubiquitination of mGluR5, which was alleviated when CAL was overexpressed. The binding between mGluR5 and CAL was also reduced by rotenone treatment while CAL overexpression recovered it (Fig. 9A). Notably, overexpression of CAL albeit altered its interaction with mGluR5, did not impact the ubiquitination of mGluR5 in the absence of rotenone treatment, indicating that the suppressed ubiquitination is dependent on rotenone-induced neurotoxicity. We then assessed the expression of mGluR5 in CAL-positive cells by confocal microscopy. The result showed that rotenone injection decreased the fluorescence intensity of mGluR5 in CALpositive cells while CAL overexpression attenuated this effect (Fig. 9B). These data suggest that CAL intercepts mGluR5 ubiquitination to enhance mGluR5 expression via interacting with the receptor in response to rotenone treatment.

In order to validate that the beneficial function of CAL depends on regulating mGluR5, MPEP was introduced to the system of AAV-mediated CAL deficiency (Fig. 9C) and the protein level of CAL was knocked down as shown in Fig. 9D. In the AAV-scramble group, MPEP reduced hemilateral turnings in the apomorphine-induced rotational behavior, and attenuated $\mathrm{TH}$-positive neurons against rotenone lesion, which is consistent with previously reported studies [4, 44], while CAL deficiency partially disrupted the protective effect of MPEP (Fig. 9E and F). Furthermore, compared to the observation in the rotenone-treated group, MPEP also upregulated protein expression of mGluR5 and TH, and decreased JNK phosphorylation induced by rotenone treatment, while CAL 
a

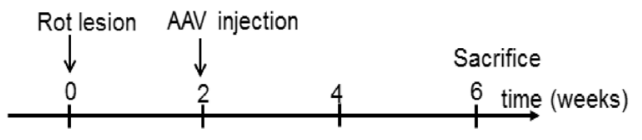

b
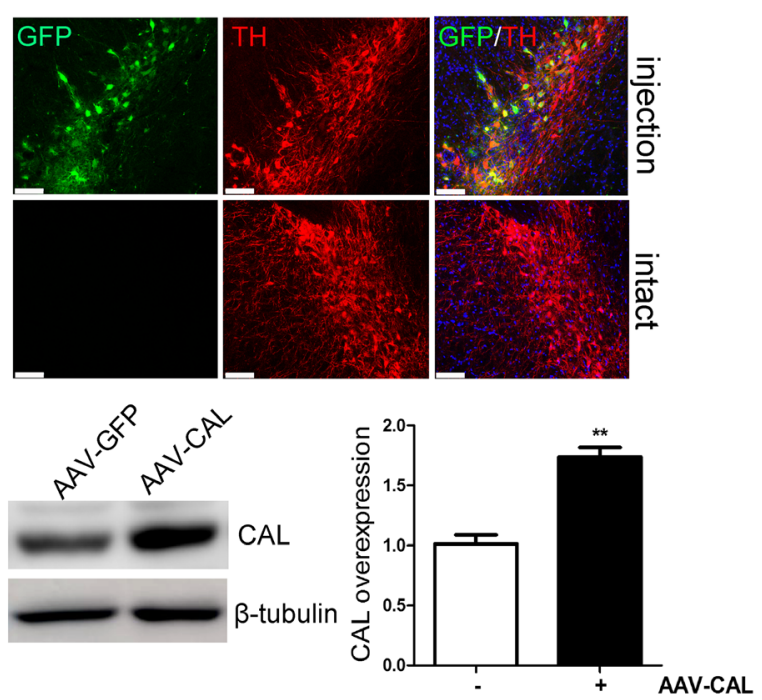

d
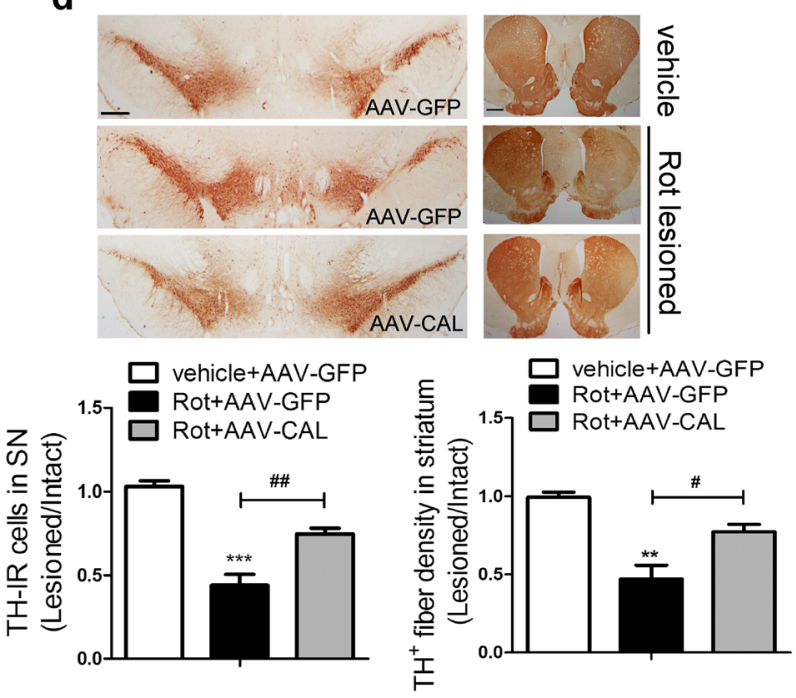

f
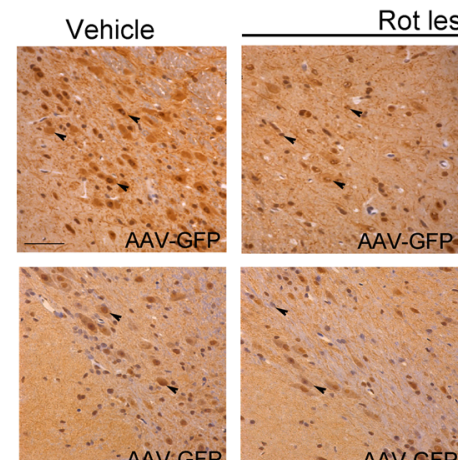

Rot lesioned
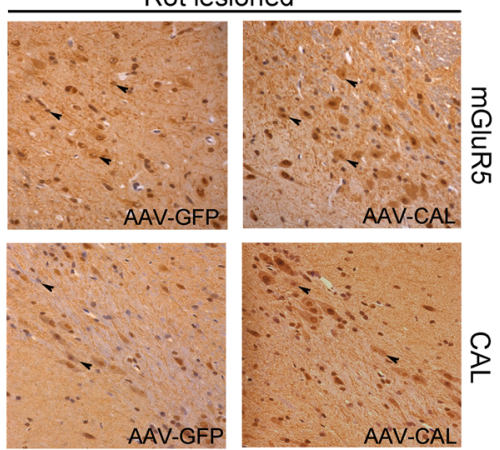

C
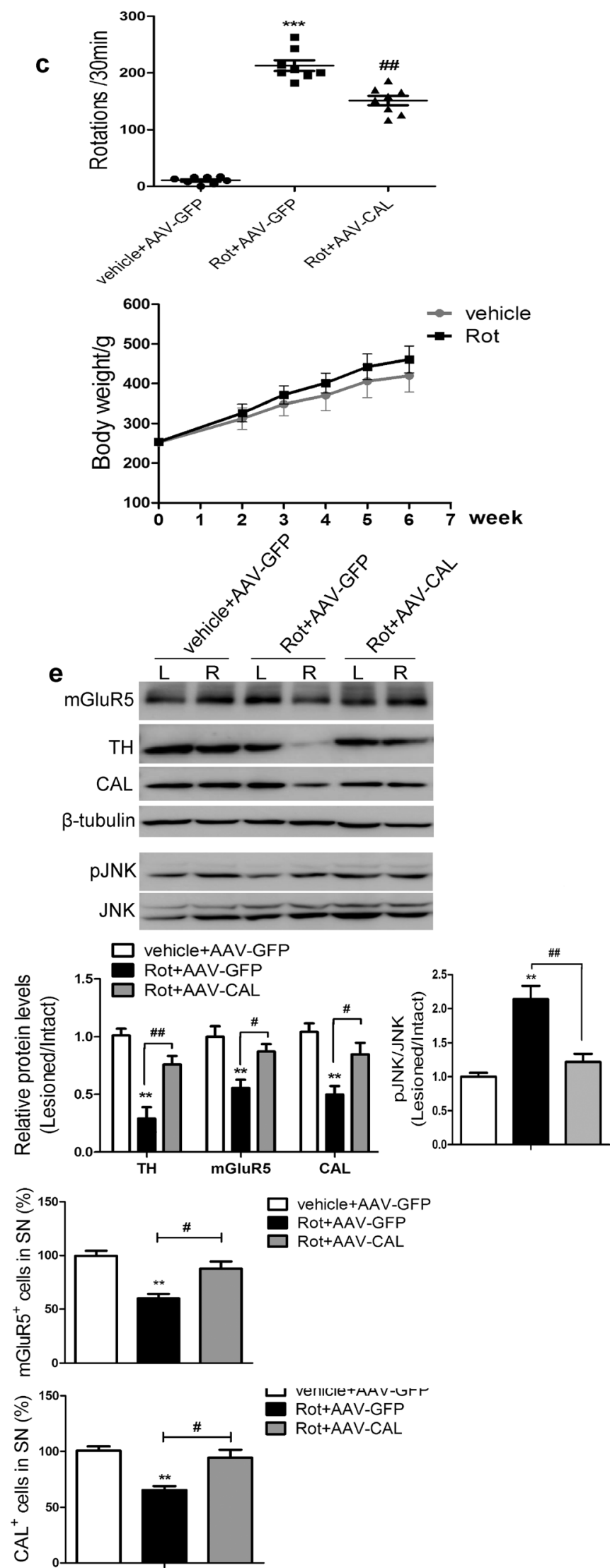
4 Fig. 7 CAL overexpression upregulates mGluR5 expression to protect against cell death in the rotenone-induced animal model of PD. (A) The treatment of rats is shown in the scheme. (B) At 4 weeks after AAV injection into the SN of animals, GFP and TH were immuno-labeled (red) and images were captured by confocal microscopy (up), and the expression of CAL in SN tissues was detected by Western blotting (down). Scale bar $=75 \mu \mathrm{m}$. The protein level was normalized to $\beta$ tubulin and represented as the fold difference of the AAV-GFP group ( $n=3$ or 4 /group). (C) Following virus injection, animal behavioral asymmetry with the apomorphine-induced rotation test and the total hemilateral rotations within $30 \mathrm{~min}$ were evaluated (up) ( $n=8 /$ group). Body weight each week during surgery was measured (down) $(n=8 /$ group). (D) TH-positive immunoreactive neurons in the $\mathrm{SN}$ and TH-IR fibers in the striatum were detected by immunohistochemistry. Scale bar = $500 \mu \mathrm{m}(\mathrm{SN}), 1 \mathrm{~mm}$ (striatum). Quantification of TH-positive immunoreactivity was represented by the ratio of lesion side to intact side $(n=3$ or 4/group). (E) Brain lysates from the left (L) and right (R) striatum were analyzed by Western blotting to observe the alteration in mGluR5, CAL, and $\mathrm{p}$-JNK protein levels. Protein levels were normalized to $\beta$-tubulin and represented as the ratio of lesion side to intact side and expressed as the fold difference of the control group ( $n=3$ or 4/group). (F) mGluR5 and CAL staining in SN sections were detected by immunohistochemistry (left) and the number of positive cells was analyzed (right). The quantification represented the percentage in the control group. The black arrows represented positive cells that were stained brown (scale bar $=$ $50 \mu \mathrm{m})$. Data shown in all panels in this figure represent the mean \pm $\mathrm{SD}$. The statistical significance was determined using Student's $t$ test (B) or 1-way ANOVA followed by Dunnett's test (C-F). Vehicle groups with AAV-GFP infection were used as controls. $* * p<0.01, * * * p<0.001$ versus control, ${ }^{\#} p<0.05,{ }^{\# \#} p<0.01$ compared to the rotenone lesion with AAV-GFP delivery group

knockdown blocked it (Fig. 9G and H). To this end, these data illustrate that CAL plays a significant role to resist rotenoneinduced behavioral deficit and dopaminergic neuronal death in vivo, which is attributed to the modulation of mGluR5 expression and activity through their interaction.

\section{Discussion}

Excitotoxicity due to overstimulation of glutamate receptors has been reported to be closely associated with the progression of several neurodegenerative diseases such as PD among others [42]. mGluR5 has been proposed as a potential target for the pharmacological treatment of PD [45], due to its widespread distribution within the basal ganglia [46]. Blockade of mGluR 5 by the selective antagonist MPEP/MTEP or NAMs produces anti-parkinsonian-like effects in PD animal models [47-50] and in clinical trials [51-53], suggesting that regulation of mGluR5 stands as a promising strategy in controlling the progression of PD. In the present study, we found that CAL enhanced the protection against STS-induced apoptosis through group I mGluRs-mediated AKT and ERK1/2 in C6 astroglial cells. Importantly, in a rotenone-induced cellular model of PD, CAL mitigated glutamate excitotoxicity, upregulated the protein level of mGluR5 and downregulated the JNK pathway to consequently inhibit cell death. CAL consistently ameliorated motor dysfunction and TH-positive cell loss in the nigrostriatal pathway in rotenone-treated animals.

The interacting proteins, such as adenosine receptor $\mathrm{A}_{2 \mathrm{~A}}$ or $\mathrm{N}$-methyl-D-aspartic acid (NMDA) receptor, regulate mGluR5 function, and simultaneous blocking of these proteins and mGluR5 results in the reciprocal synergistic therapeutic effect in PD $[54,55]$. Moreover, dopaminergic degeneration in PD models results in increased glutamate release in the dorsal striatum $[56,57]$, and blockade of this excess excitatory drive may alternatively modulate excitatory transmission in the basal ganglia to attenuate parkinsonian symptoms [5]. This evidence suggests that the identification of the regulatory factors to target mGluR5 might provide a novel therapeutic strategy for PD. In the present study, we found that rotenone increased the level of glutamate and decreased mGluR5 protein expression. CAL, as the interacting partner of mGluR5, exerted a protective role by upregulating mGluR5 protein expression and decreasing glutamate release to mitigate excitotoxicity. Furthermore, the inhibited rotenoneinduced mGluR5 degradation by overexpression of CAL was blocked by treatment with its agonist DHPG, possibly due to receptor overactivation (Fig. 6). MPEP inhibited mGluR5 overactivation leading to mGluR5 protein increase and glutamate decrease while knockdown of CAL reduced these effects (Figs. 6 and 9). These findings indicate that the decreased protein level of mGluR5 in this rotenone-induced PD model may be as a result of an adaptive mechanism in response to excitotoxicity, and CAL may act as an appropriate modulator for fine-tuning of mGluR5 activation. In addition, extracellular glutamate is removed by cellular uptake, which is reportedly mediated by $\mathrm{Na}^{+}$- and $\mathrm{K}^{+}$-dependent transporters such as excitatory amino acid transporters [58], whereas glutamate release is due to exocytosis of synaptic vesicles or glutamate transporters, and cysteine transporters [58]. Whether these glutamate regulatory systems are involved in the inhibition of CAL at the rotenone-induced extracellularincreased level of glutamate requires further investigation.

Previously reported studies reveal that mGluR5 mRNA in the striatum is decreased (rostral and caudal) in a reserpinized PD model [59] and in 6-OHDA MFB-lesioned rats [60], while an increase in mGluR5 protein expression in the striatum is observed in the dopaminergic degenerative system induced by 6-OHDA and MPTP-lesioned PD animal models [61-63]. Here, we showed a marked decrease in mGluR5 protein expression in a rotenone-lesioned rat model. These observations suggest that differential regulation of mGluR 5 mediated by certain factors may occur depending on different stimuli. mGluR5 activation has consistently been found to support neuronal survival in cultured cerebellar granule cells [64], and activation of mGluRs increases $\beta$-amyloid precursor protein release, thereby reducing neurotoxicity in Alzheimer's disease [65]. In contrast, overstimulation by glutamate 

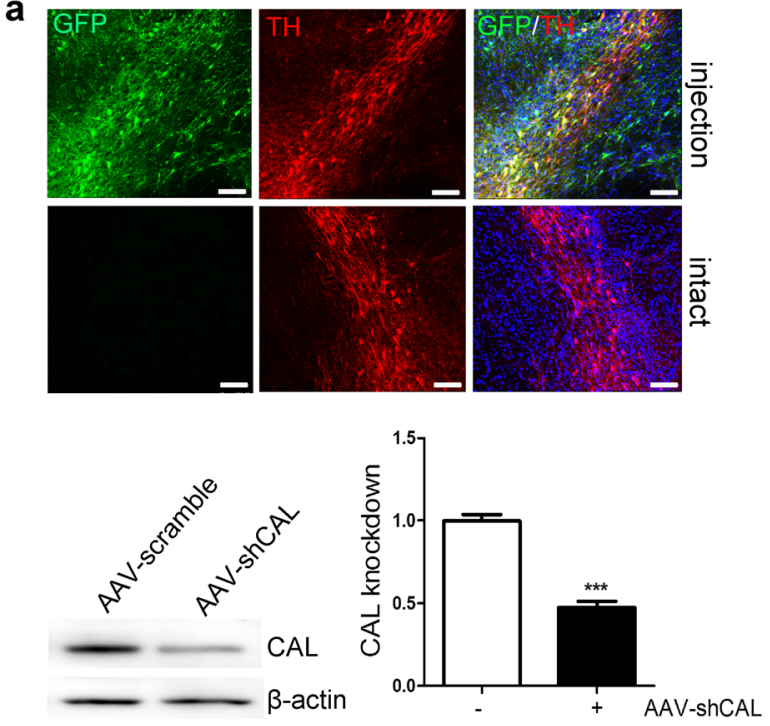

C
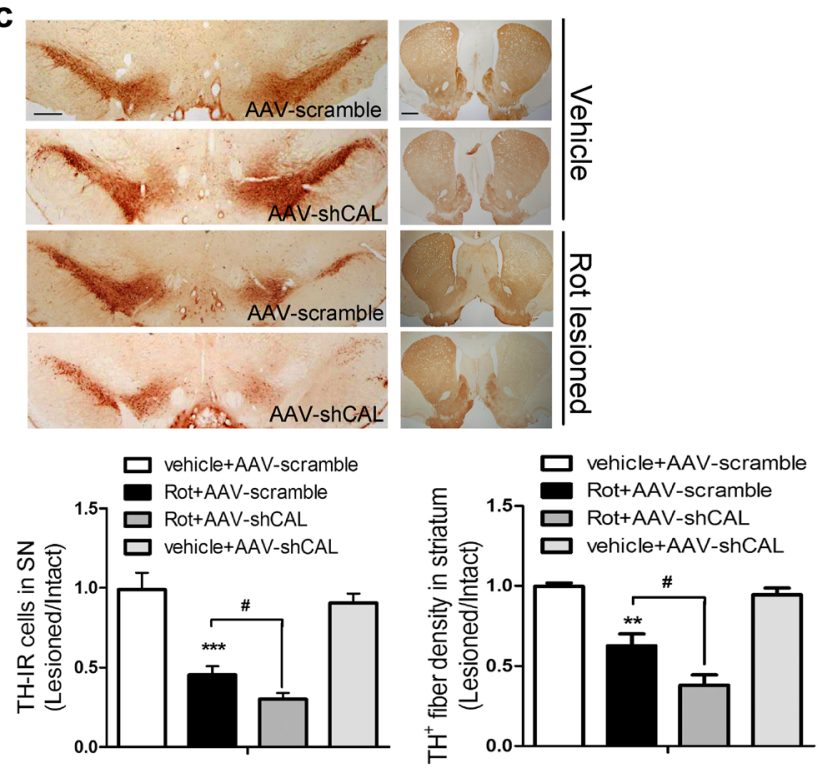

b
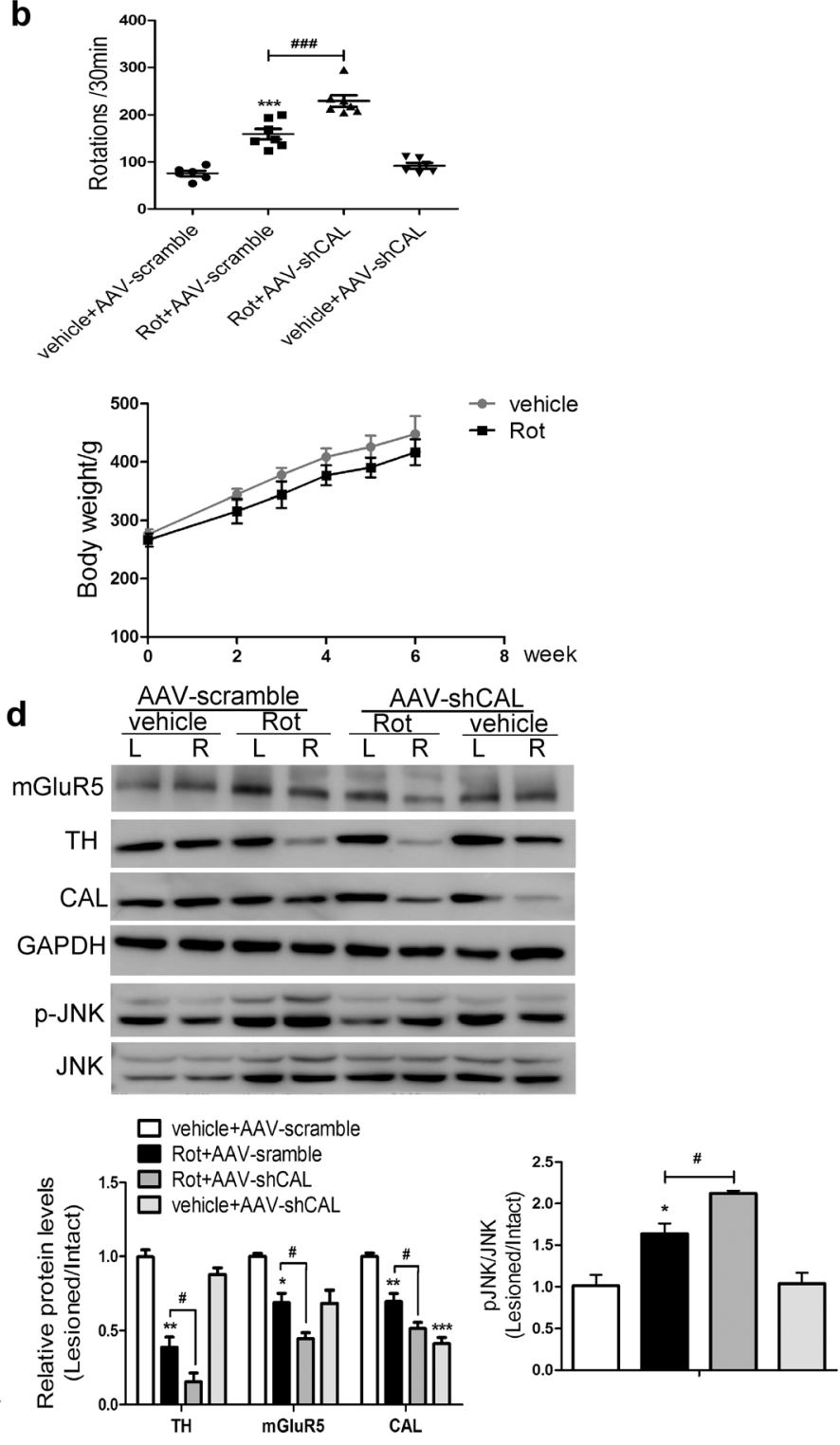

e
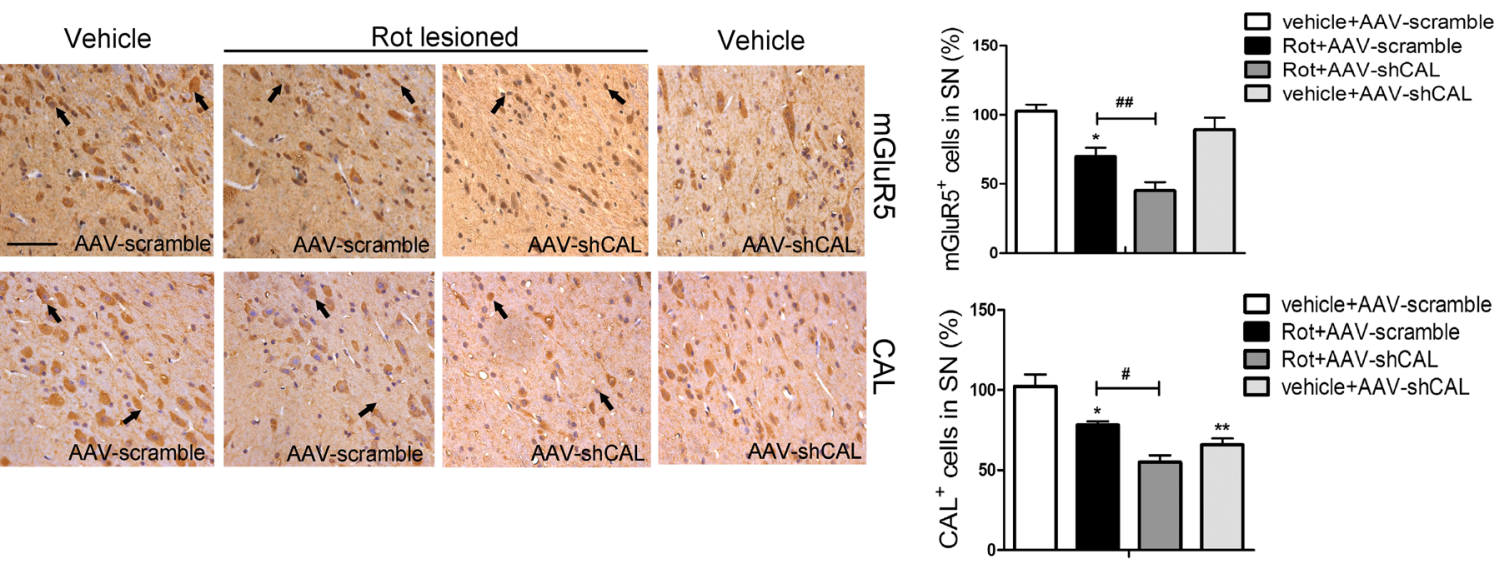

produces excitotoxicity and blockade of mGluR5 by the selective antagonist MPEP or MTEP produces antiparkinsonian-like effects in PD animal models [66, 67].
Therefore, it appears mGluR5 may have a dual action, by which activation of the receptor by an agonist exerts protection against cell death, while blockade of mGluR5 by a 
4 Fig. 8 Effect of CAL deficiency on rotenone-induced toxicity in vivo. (A) Following 4 weeks of AAV delivery, the $\mathrm{SN}$ sections were subjected to TH labeling (red) and the images with GFP immunofluorescence were obtained by confocal microscopy (up). Scale bar $=100 \mu \mathrm{m}$. The interference efficiency of AAV-shCAL was investigated by Western blotting (down, left) and quantification analysis was represented as the fold difference of the AAV-scramble group (down, right). (B) After virus injection, the animals underwent the apomorphine-induced rotational test to assess behavioral asymmetry. The total number of hemilateral rotational turns within 30 min was evaluated (up) ( $n=7 /$ group) and the body weight during surgery was monitored (down) ( $n=7 /$ group). (C) THpositive immunoreactivity was assessed by immunohistochemistry in the $\mathrm{SN}$ and striatum. Scale bar $=500 \mu \mathrm{m}(\mathrm{SN}), 1 \mathrm{~mm}$ (striatum). Quantification of TH-IR neurons in the SN or TH-IR fibers in the striatum represented the ratio of lesion side to intact side ( $n=3$ or 4/group). (D) The alterations in mGluR5, CAL, and p-JNK in the left (L) and right (R) striatum were assessed by Western blotting (up). Protein levels were normalized to GAPDH (TH, mGluR5, CAL) or total JNK (p-JNK) and represented as the ratio of lesion side to intact side by the expression of fold difference of the control group ( $n=3 /$ group). (E) mGluR5- and CAL-positive immunoreactivity in the SN was analyzed by immunohistochemistry (left) and the number of positive cells was analyzed (right). The quantification represented the percentage in the control group $(n=3$ or 4/group). The black arrows represent positive cells that were stained brown (scale bar $=50 \mu \mathrm{m}$ ). Vehicle groups with AAV-scramble virus delivery were used as controls. Data shown in all panels of this figure represent the mean $\pm \mathrm{SD}$. Statistical significance was determined using Student's $t$ test (A) or 1-way ANOVA followed by Dunnett's test (B-E). ${ }^{*} p<0.05, * * p<0.01, * * * p<0.001$ versus control, ${ }^{*} p<0.05,{ }^{\# \#} p<0.01$, \#\#\# $p<0.001$ compared to the rotenone lesion with AAV-scramble delivery group

selective antagonist inhibits the receptor hyperactivity. Indeed, in our study, CAL protected against apoptosis by facilitating DHPG-activated mGluR5 following STS treatment in C6 astroglial cells. In the PD model, overactivation of mGluR5 by rotenone caused cell apoptosis, and correspondingly reduced both the expression of mGluR 5 and CAL by weakening the association between these 2 proteins, which was worsened by CAL knockdown and restored by CAL overexpression. Our findings suggest that blockade of mGluR5 inhibits rotenone-induced receptor overactivation by reducing the release of glutamate to further restore the normal expression of the receptor, subsequently producing anti-parkinsonian-like effects in PD. Correspondingly, CAL may interact with mGluR5 to modulate its expression to exert its protective function depending on the needs of the cells. In addition, CAL played a protective role by upregulating mGluR 5 protein expression at the post-translational level by inhibiting the ubiquitin-proteasome pathway. It was previously reported that mGluR5 can be ubiquitinated by 7 in absentia homolog $1 \mathrm{~A}$ (Siah1A), a member of the RING-finger-containing E3 ubiquitin ligases, by binding to the Siah-interacting domain at the C-terminal tails of mGluR5 [39]. The CAL-mGluR5 association inhibited mGluR5 ubiquitination and this association possibly spatially obstructed the interaction of Siahla and mGluR5 which prevented mGluR5 ubiquitination. However, this possibility requires further investigation and what controls the rotenone-induced reduction of CAL protein level also remains unclear.

The activation of group I mGluRs modulates PI3K and increases AKT phosphorylation to inhibit neuronal apoptosis [68]. The JNK signaling activation thought to precede apoptosis is related to dopaminergic neuronal loss in neurotoxicantand environmental stress-induced PD models [40]. Here, we found that CAL inhibited STS-induced cell apoptosis by increasing group I mGluRs-activated AKT and ERK1/2 signaling pathways in $\mathrm{C} 6$ astroglial cells. In the rotenone-induced model of PD, overexpression of CAL inhibited cell apoptosis through mGluR5-mediated phosphorylation of JNK, which was disrupted when the receptor was profoundly activated by DHPG treatment. Our study indicates that the association between CAL and mGluR5 controls rotenone-induced receptor overactivation by decreasing the level of glutamate and upregulating the protein level of mGluR5 through ubiquitination-proteasome-mediated mGluR5 degradation, and further mediates the related signaling pathway to exert neuroprotection against cell apoptosis (Fig. 10). This might be that the environment of reactive oxygen species (ROS) generated by rotenone [69] decreased the expression of CAL, which further interfered with mGluR5 interaction and its intracellular binding partners, ultimately leading to the reduction of receptor surface localization. This study also suggests that compared to NAMs, targeting the endogenous molecule of PDZ domain-containing protein CAL may act as an appropriate modulator for fine-tuning mGluR5 activation to promote its therapeutic efficacy in the treatment of PD. In addition, D1 and D2 receptors are abundantly expressed in the basal ganglia and play a critical role in regulating the physiological effect of dopamine in PD [70], while D2 receptor forms a hetero-receptor complex with mGluR5 and the $\mathrm{A}_{2 \mathrm{~A}}$ receptor in dendritic spines of striatopallidal neurons [71]. Collectively, it would be interesting to examine how these factors mentioned above are involved in contributing to the beneficial effect of CAL against dopaminergic neuronal death by regulating mGluR5 in PD.

Interestingly, CAL exerted its protective effect on mGluR5 function depended on the presence of rotenone. Knockdown of CAL without rotenone treatment did not cause a decrease in the level of mGluR5 in MN9D cells (Fig. 3F and G). Correspondingly, we did not observe any of the behavioral deficits when injecting shCAL in SN in the animal group without rotenone treatment (Fig. 8B). However, we observed the interactive change between CAL and mGluR5 but the receptor ubiquitination was not affected after CAL overexpression (Fig.4D, lanes 4 versus 1; Fig. 9A, lanes 8 versus 7). It might be speculated that, compared to the pathogenesis in $\mathrm{PD}$, under a given physiological condition, CAL interaction with mGluR5 in dopaminergic neurons may not be involved in the regulation of mGluR5 protein degradation but, perhaps, in other 
a
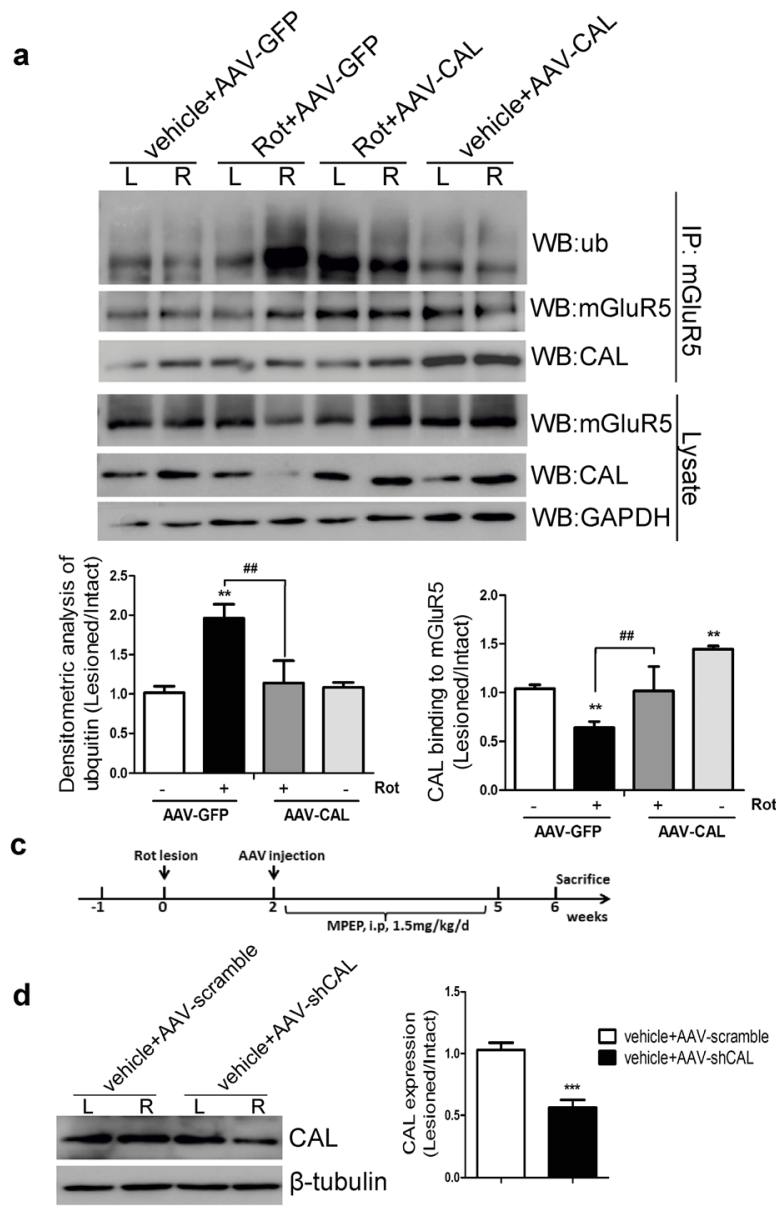

e

$\otimes$ vehicle+AAV-scramble

Rot+AAV-scramble

血 Rot+AAV-scramble+MPEP

Rot+AAV-shCAL+MPEP

vehicle+AAV-shCAL
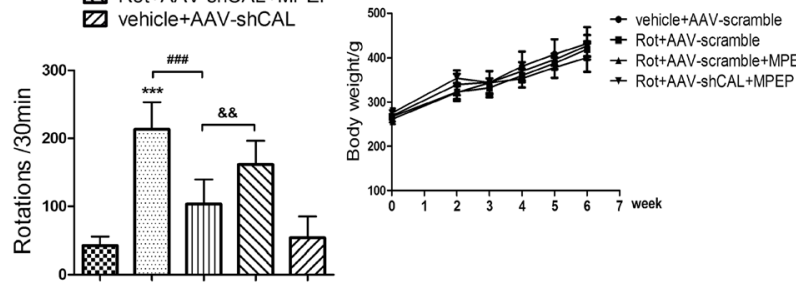

- Rot+AAV-scramble+MPEP
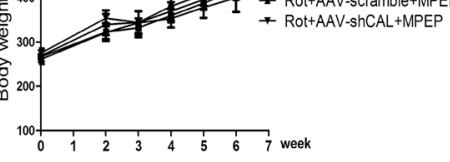

${ }_{0}$ 这.

g

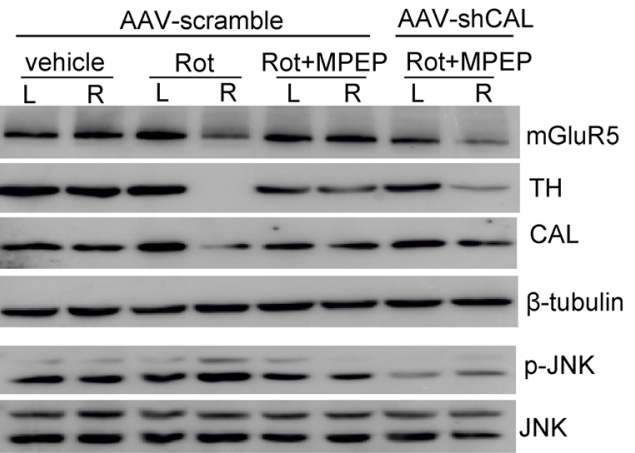

h b
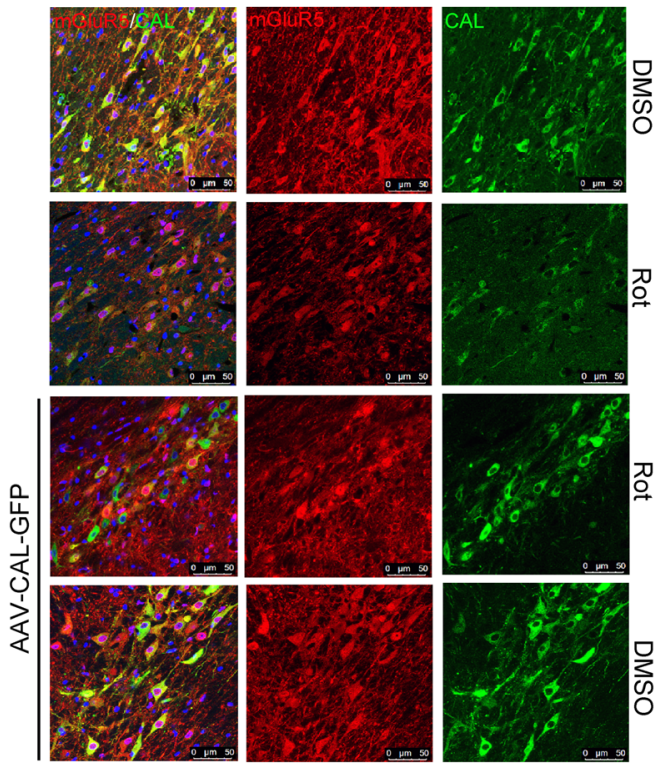

$\frac{\frac{0}{0}}{\frac{0}{2}}$
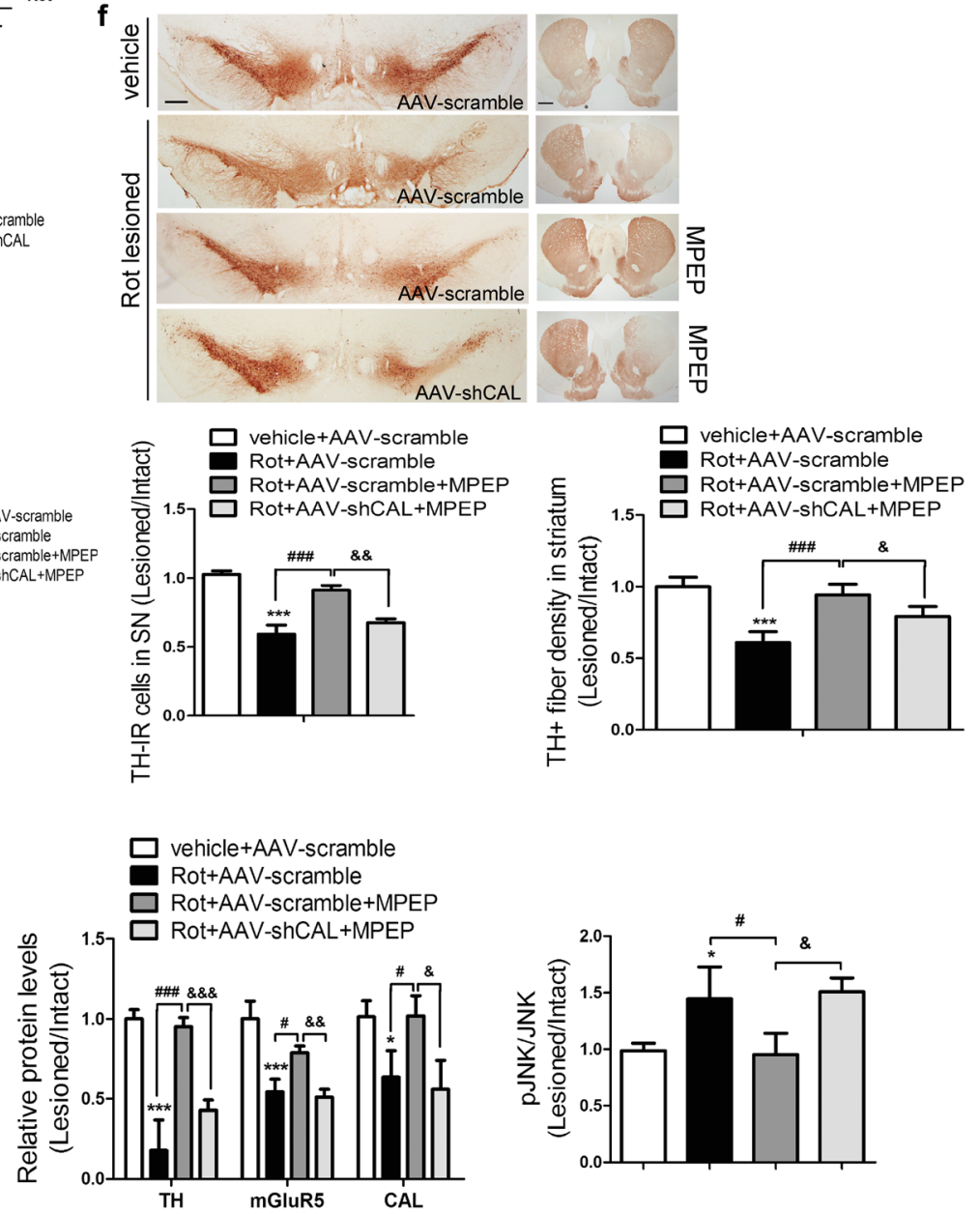

cellular-intrinsic functions. Notably, in C6 astroglial cells, knockdown of CAL without mGluR5 activation by DHPG showed the opposite effect compared to that with DHPG treatment (Figs. 1D and $2 \mathrm{C}$ and $\mathrm{D}$, lanes 4 versus 2 ), which increased the phosphorylation of ERK $1 / 2$ and AKT resulting in the inhibition of apoptosis, suggesting that 
Fig. 9 CAL regulated mGluR5 degradation through the interaction and deficiency of CAL abrogated the beneficial effect of MPEP in vivo. (A) The brain tissues were lysed for coimmunoprecipitation. Lysates were first incubated with mGluR5 antibody followed by protein-G beads to immunoprecipitate; the complex was detected with anti-ubiquitin or antiCAL antibodies by Western blot to test mGluR5 ubiquitination and interaction of CAL (up). The whole lysates were detected with mGluR5 and CAL antibodies to visualize the expression (down). Protein levels were normalized to immunoprecipitated mGluR5 and represented as the fold different ratio (right/left) of the control group. (B) Immunofluorescence of mGluR5 (red) and CAL (green) in the SN section showing the protein expression and colocalization. Scale bar $=50 \mu \mathrm{m}$. (C) Rats were lesioned by rotenone for 2 weeks followed by CAL knockdown and MPEP administered for 3 weeks as the process shown. (D) The interference efficiency of AAV-shCAL was detected by Western blotting $(n=3$, left) and quantification analysis was represented as the ratio of lesion side to intact side by the fold difference of the AAV-scramble group (right). (E) The body weight and apomorphine-induced rotational behavior were monitored ( $n=6-8$ /group). (F) TH-positive immunoreactivity in SN and striatum was detected by immunohistochemistry and quantification represented as the ratio of lesion side to intact side $(n=3)$. Scale bar $=500 \mu \mathrm{m}$ (SN), $1 \mathrm{~mm}$ (striatum). $(\mathrm{G}, \mathrm{H})$ The expression of mGluR5, CAL, TH, and $\mathrm{p}$-JNK were detected by Western blot. Protein levels were normalized to $\beta$-tubulin or total JNK and represented as the ratio of lesion side to intact side by the expression of fold difference of the control group $(n=3)$. Data shown represent the mean $\pm \mathrm{SD}$. The statistical significance was determined by 1 -way ANOVA followed by Dunnett's test. $* p<0.05$, $* * p<0.01, * * * p<0.001$ versus control, ${ }^{\#} p<0.05,{ }^{\# \#} p<0.01$, \#\#\# $p<0.001$ compared to rotenone group, ${ }^{\&} p<0.05,{ }^{\&} \&_{p}<0.01$, $\& \& \& p<0.001$ compared to rotenone group with MPEP treatment
CAL may perhaps play a role in anti-protection when it is independent of mGluR5 activity. This discrepancy may be due to the difference in cell type. Regardless of the complication of the function of CAL without mGluR5 activation that needs to be further investigated, our study demonstrates the protective role of CAL following toxin stimulation by regulating mGluR5 activity.

\section{Summary}

Our study indicates that CAL coupled with mGluR5 upregulates mGluR5 protein expression by inhibition of ubiquitin-proteasome-dependent degradation and modulates receptor-mediated downstream AKT, ERK1/2, and JNK signaling pathways, subsequently protecting against cell apoptosis. This study provides a novel mechanism by which CAL acts as a target to regulate cell apoptosis via the regulation of mGluR5, which may open the way for future discoveries of therapeutic strategy for the treatment of PD.

Acknowledgements This study was supported by the National Natural Science Foundation of the People's Republic of China (81171886; 81372587), Beijing Municipal Natural Science Foundation (No. 7132018), Beijing City Board of Education Development Project (KZ201310025021), and the Project of Construction of Innovative Teams and Teacher Career Development for Universities and Colleges Under Beijing Municipality (No. IDHT20140514). We thank
Fig. 10 Schematic graph of the neuroprotective role of scaffold protein CAL via regulation of mGluR5. The association of CAL with mGluR5 controlled rotenone-induced receptor overactivation by decreasing the level of glutamate and $\mathrm{u}-$ regulating the protein level of mGluR5 through ubiquitinationproteasome-mediated mGluR5 degradation, and further mediation of the related signaling pathway to exert neuroprotection against cell apoptosis

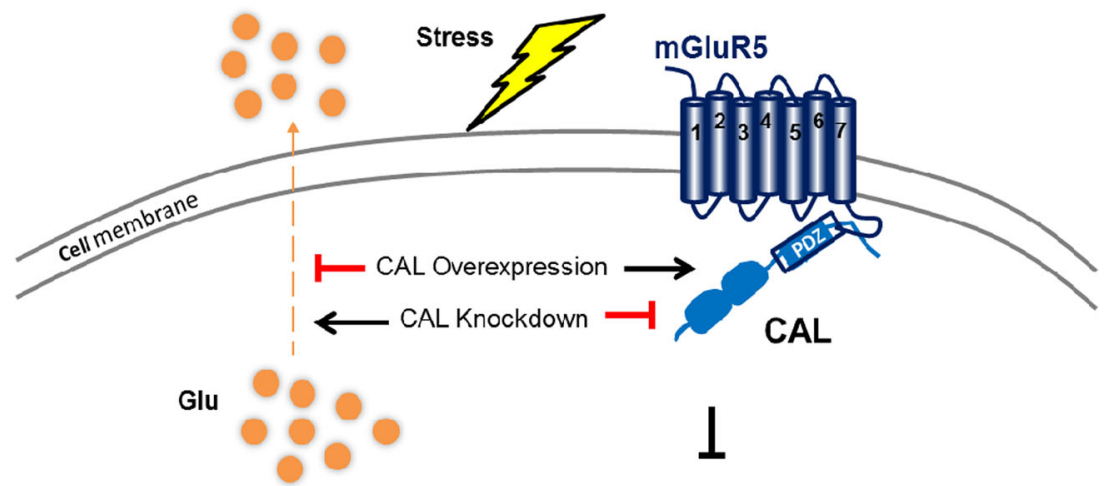

mGluR5 degradation

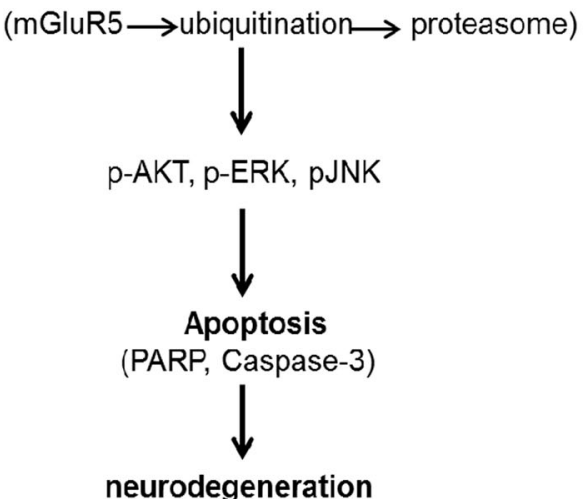


International Science Editing (http://www.internationalscienceediting. com) for editing this manuscript. The authors thank all members in the group for helpful suggestions and discussion.

\section{References}

1. Dauer W, Przedborski S. Parkinson's disease: mechanisms and models. Neuron. 2003; 39: 889-909.

2. DeLong MR, and Wichmann T. Basal Ganglia Circuits as Targets for Neuromodulation in Parkinson Disease. JAMA Neurol. 2015; 72: $1354-1360$.

3. Pin JP, and Duvoisin R. The metabotropic glutamate receptors: structure and functions. Neuropharmacology. 1995; 34: 1-26.

4. Hsieh M, Ho S, Yeh K, et al. Blockade of metabotropic glutamate receptors inhibits cognition and neurodegeneration in an MPTPinduced Parkinson's disease rat model. Pharmacol Biochem Behav. 2012; 102: 64-71.

5. Gasparini F, Di Paolo T, and Gomez-Mancilla B. Metabotropic Glutamate Receptors for Parkinson's Disease Therapy. Parkinson's Dis. 2013; 2013: 196028.

6. Litim N, Morissette M, and Di Paolo T. Metabotropic glutamate receptors as therapeutic targets in Parkinson's disease: An update from the last 5 years of research. Neuropharmacology. 2017; 115: $166-179$.

7. Vernon AC, Zbarsky V, Datla KP, Croucher M J, and Dexter DT. Subtype selective antagonism of substantia nigra pars compacta Group I metabotropic glutamate receptors protects the nigrostriatal system against 6-hydroxydopamine toxicity in vivo. J Neurochem. 2007; 103: 1075-1091.

8. Morin N, Grégoire L, Morissette M, et al. MPEP, an mGlu5 receptor antagonist, reduces the development of 1-DOPA-induced motor complications in de novo parkinsonian monkeys: Biochemical correlates. Neuropharmacology. 2013; 66: 355-364.

9. Duvey G, Perry B, Le Poul E, et al. A novel series of metabotropic glutamate receptor 5 negative allosteric modulators based on a 4, 5 , 6, 7- tetrahydropyrazolo [1, 5-a] pyridine core. Bioorg Med Chem Lett. 2013; 23: 4523-4527.

10. Rascol O, Fox S, Gasparini F, Kenney C, Di Paolo T, and GomezMancilla B. Use of metabotropic glutamate 5-receptor antagonists for treatment of levodopa-induced dyskinesias. Parkinsonism Relat Disord. 2014; 20: 947-956.

11. Emmitte KA. mGlu5 negative allosteric modulators: a patent review (2013 - 2016). Expert Opin Ther Pat. 2017; 27: 691-706.

12. Niswender CM, and Conn PJ. Metabotropic Glutamate Receptors: Physiology, Pharmacology, and Disease. Annu Rev Pharmacol. 2010; 50: 295-322.

13. Tu JC, Xiao B, Naisbitt $\mathrm{S}$, et al. Coupling of mGluR/Homer and PSD-95 Complexes by the Shank Family of Postsynaptic Density Proteins. Neuron. 1999; 23: 583-592.

14. Kitano J, Kimura K, Yamazaki Y, et al. Tamalin, a PDZ DomainContaining Protein, Links a Protein Complex Formation of Group 1 Metabotropic Glutamate Receptors and the Guanine Nucleotide Exchange Factor Cytohesins. J Neurosci. 2002; 22: 1280-1289.

15. Paquet M, Asay MJ, Fam SR, et al. The PDZ Scaffold NHERF-2 Interacts with mGluR5 and Regulates Receptor Activity. J Biol Chem. 2006; 281: 29949-29961.

16. Neudauer CL, Joberty G, and Macara IG. PIST: a novel PDZ/ coiled-coil domain binding partner for the rho-family GTPase TC10. Biochem Biophys Res Commun. 2001; 280: 541-547.

17. Charest A, Lane K, McMahon K, and Housman DE. Association of a novel PDZ domain-containing peripheral Golgi protein with the Q-SNARE (Q-soluble N-ethylmaleimide-sensitive fusion protein
(NSF) attachment protein receptor) protein syntaxin 6. J Biol Chem. 2001; 276: 29456-29465.

18. Cheng J, Moyer B D, Milewski M, Loffing J, Ikeda M, Mickle J E, Cutting G R, Li M, Stanton B A, Guggino W B. A Golgi-associated PDZ domain protein modulates cystic fibrosis transmembrane regulator plasma membrane expression. J Biol Chem, 2002, 277: 3520-3529.

19. Cheng J, Wang H, Guggino W B. Modulation of mature cystic fibrosis transmembrane regulator protein by the PDZ domain protein CAL. J Biol Chem, 2004, 279: 1892-1898.

20. He J, Bellini M, Xu J, Castleberry A M, Hall R A. Interaction with cystic fibrosis transmembrane conductance regulator-associated ligand (CAL) inhibits beta1-adrenergic receptor surface expression. $\mathrm{J}$ Biol Chem, 2004, 279: 50190-50196.

21. Wente W, Efanov A M, Treinies I, Zitzer H, Gromada J, Richter D, Kreienkamp H. The PDZ/coiled-coil domain containing protein PIST modulates insulin secretion in MIN6 insulinoma cells by interacting with somatostatin receptor subtype 5. FEBS Letters, 2005, 579: 6305-6310.

22. Wente W, Stroh T, Beaudet A, Richter D, Kreienkamp H J. Interactions with PDZ domain proteins PIST/GOPC and PDZK1 regulate intracellular sorting of the somatostatin receptor subtype 5. J Biol Chem, 2005, 280: 32419-32425.

23. Xu Z, Oshima K, Heller S. PIST regulates the intracellular trafficking and plasma membrane expression of cadherin 23. BMC Cell Biol, 2010, 11: 80.

24. Lu R, Stewart L, Wilson J M. Scaffolding protein GOPC regulates tight junction structure. Cell and Tissue Research, 2015, 360: 321-332.

25. Ito H, Iwamoto I, Morishita R, Nozawa Y, Asano T, Nagata K. Identification of a PDZ protein, PIST, as a binding partner for Rho effector Rhotekin: biochemical and cell-biological characterization of Rhotekin-PIST interaction. Biochemical J, 2006, 397: 389-398.

26. Ito H, Iwamoto I, Mizutani K, Morishita R, Deguchi T, Nozawa Y, Asano T, Nagata K. Possible interaction of a Rho effector, Rhotekin, with a PDZ-protein, PIST, at synapses of hippocampal neurons. Neurosci Res, 2006, 56: 165-171.

27. Chen A, Gößling EK, Witkowski L, et al. Regional and subcellular distribution of the receptor-targeting protein PIST in the rat central nervous system. J Comp Neurol, 2012; 520: 889-913.

28. Orr H T. Lurcher, nPIST, and autophagy. Neuron, 2002, 35: 813814.

29. Yue Z, Horton A, Bravin M, DeJager P L, Selimi F, Heintz N. A novel protein complex linking the delta 2 glutamate receptor and autophagy: implications for neurodegeneration in lurcher mice. Neuron, 2002, 35: 921-933.

30. Cuadra A E. AMPA Receptor Synaptic Targeting Regulated by Stargazin Interactions with the Golgi-Resident PDZ Protein nPIST. J Neurosci, 2004, 24: 7491-7502.

31. Zhang J, Cheng S, Xiong Y, et al. A novel association of mGluRla with the PDZ scaffold protein CAL modulates receptor activity. FEBS Lett. 2008; 582: 4117-4124.

32. Cheng S, Zhang J, Zhu P, et al. The PDZ domain protein CAL interacts with mGluR5a and modulates receptor expression. J Neurochem. 2010; 112: 588-598.

33. Brabet I, Mary S, Bockaert J, and Pin JP. Phenylglycine derivatives discriminate between mGluR1- and mGluR5-mediated responses. Neuropharmacology. 1995; 34: 895-903.

34. Sun L, Gu L, Wang S, et al. N-acetylcysteine protects against apoptosis through modulation of group I metabotropic glutamate receptor activity. PLoS One. 2012; 7: e32503.

35. Zhang Z, Hu F, Liu Y, et al. Activation of type 5 metabotropic glutamate receptor promotes the proliferation of rat retinal progenitor cell via activation of the PI-3-K and MAPK signaling pathways. Neuroscience. 2016; 322: 138-151.

36. Ribeiro FM, Vieira LB, Pires RGW, Olmo RP, and Ferguson SSG. Metabotropic glutamate receptors and neurodegenerative diseases. Pharmacol Res. 2017; 115: 179-191. 
37. Sherer TB, Betarbet R, Stout AK, et al. An in vitro model of Parkinson's disease: linking mitochondrial impairment to altered alpha-synuclein metabolism and oxidative damage. J Neurosci. 2002; 22: 7006-7015.

38. Bové J, Perier C. Neurotoxin-based models of Parkinson's disease. Neuroscience, 2012, 211: 51-76.

39. Moriyoshi K, Iijima K, Fujii H, Ito H, Cho Y, and Nakanishi S. Seven in absentia homolog $1 \mathrm{~A}$ mediates ubiquitination and degradation of group 1 metabotropic glutamate receptors. Proc. Natl. Acad. Sci. U S A. 2004; 101: 8614-8619.

40. Peng J, and Andersen JK. The role of c-Jun N-terminal kinase (JNK) in Parkinson's disease. IUBMB Life. 2003; 55: 267-271.

41. Wang S, Zhang C, Sheng X, Zhang X, Wang B, and Zhang G. Peripheral expression of MAPK pathways in Alzheimer's and Parkinson's diseases. J Clin Neurosci. 2014; 21: 810-814.

42. Ambrosi G, Cerri S, and Blandini F. A further update on the role of excitotoxicity in the pathogenesis of Parkinson's disease. J Neural Transm (Vienna). 2014; 121: 849-859.

43. Rodrigues RJ, Alfaro TM, Rebola N, Oliveira CR, and Cunha RA. Co-localization and functional interaction between adenosine A2A and metabotropic group 5 receptors in glutamatergic nerve terminals of the rat striatum. J Neurochem. 2005; 92: 433-441.

44. Ning Xia, Qian Zhang, Shu Ting Wang, et al. Blockade of metabotropic glutamate receptor 5 protects against DNA damage in a rotenone-induced Parkinson's disease model. Free Radic Biol Med. 2015; 89: 567-580.

45. Picconi B, and Calabresi P. Targeting metabotropic glutamate receptors as a new strategy against levodopa-induced dyskinesia in Parkinson's disease? Mov Disord. 2014; 29: 715-719.

46. Vallano A, Fernandez-Duenas V, Garcia-Negredo G, et al. Targeting striatal metabotropic glutamate receptor type 5 in Parkinson's disease: bridging molecular studies and clinical trials. CNS Neurol Disord Drug Targets. 2013; 12: 1128-1142.

47. Ossowska K, Konieczny J, Wolfarth S, and Pilc A. MTEP, a new selective antagonist of the metabotropic glutamate receptor subtype 5 (mGluR5), produces antiparkinsonian-like effects in rats. Neuropharmacology. 2005; 49: 447-455.

48. Morin N, Grégoire L, Gomez-Mancilla B, Gasparini F, and Di Paolo T. Effect of the metabotropic glutamate receptor type 5 antagonists MPEP and MTEP in parkinsonian monkeys. Neuropharmacology. 2010; 58: 981-986.

49. Grégoire L, Morin N, Ouattara B, et al. The acute antiparkinsonian and antidyskinetic effect of AFQ056, a novel metabotropic glutamate receptor type 5 antagonist, in L-Dopa-treated parkinsonian monkeys. Parkinsonism Relat Disord. 2011; 17: 270-276.

50. Bezard E, Pioli EY, Li Q, et al. The mGluR5 negative allosteric modulator dipraglurant reduces dyskinesia in the MPTP macaque model. Mov Disord. 2014; 29: 1074-1079.

51. Berg D, Godau J, Trenkwalder C, et al. AFQ056 treatment of levodopa-induced dyskinesias: Results of 2 randomized controlled trials. Mov Disord. 2011; 26: 1243-1250.

52. Stocchi F, Rascol O, Destee A, et al. AFQ056 in Parkinson patients with levodopa-induced dyskinesia: 13-week, randomized, dosefinding study. Mov Disord. 2013; 28: 1838-1846.

53. Tison F, Keywood C, Wakefield M, et al. A Phase 2A Trial of the Novel mGluR5-Negative Allosteric Modulator Dipraglurant for Levodopa-Induced Dyskinesia in Parkinson's Disease. Mov Disord. 2016; 31: 1373-1380.

54. Coccurello R, Breysse N, and Amalric M. Simultaneous blockade of adenosine $\mathrm{A} 2 \mathrm{~A}$ and metabotropic glutamate mGlu5 receptors increase their efficacy in reversing Parkinsonian deficits in rats. Neuropsychopharmacology. 2004; 29: 1451-1461.

55. Armentero M, Fancellu R, Nappi G, Bramanti P, and Blandini F. Prolonged blockade of NMDA or mGluR5 glutamate receptors reduces nigrostriatal degeneration while inducing selective metabolic changes in the basal ganglia circuitry in a rodent model of Parkinson's disease. Neurobiol Dis. 2006; 22: 1-9.

56. Meshul CK, Emre N, Nakamura CM, Allen C, Donohue MK, and Buckman JF. Time-dependent changes in striatal glutamate synapses following a 6-hydroxydopamine lesion. Neuroscience. 1999; 88: 1-16.

57. Farrand AQ, Gregory RA, Bäckman CM, Helke KL, and Boger HA. Altered glutamate release in the dorsal striatum of the MitoPark mouse model of Parkinson's disease. Brain Res. 2016; 1651: 88-94.

58. Danbolt NC. Glutamate uptake. Prog Neurobiol. 2001; 65: 1-105.

59. Ismayilova N, Verkhratsky A, and Dascombe MJ. Changes in mGlu5 receptor expression in the basal ganglia of reserpinised rats. Eur J Pharmacol. 2006; 545: 134-141.

60. Yu T, Wang S, Liu J, and Yin H. Changes in the Gene Expression of $\mathrm{GABA}_{\mathrm{A}}$ Receptor $\alpha 1$ and $\alpha 2$ Subunits and Metabotropic Glutamate Receptor 5 in the Basal Ganglia of the Rats with Unilateral 6-Hydroxydopamine Lesion and Embryonic Mesencephalic Grafts. Exp Neurol. 2001; 168: 231-241.

61. Pellegrino D, Cicchetti F, Wang X, et al. Modulation of dopaminergic and glutamatergic brain function: PET studies on parkinsonian rats. J Nucl Med. 2007; 48: 1147-1153.

62. Sanchez-Pernaute R, Wang JQ, Kuruppu D, et al. Enhanced binding of metabotropic glutamate receptor type 5 (mGluR5) PET tracers in the brain of parkinsonian primates. Neuroimage. 2008; 42: 248-251.

63. Morin N, Morissette M, Gregoire L, Gomez-Mancilla B, Gasparini F, and Di Paolo T. Chronic treatment with MPEP, an mGlu5 receptor antagonist, normalizes basal ganglia glutamate neurotransmission in L-DOPA-treated parkinsonian monkeys. Neuropharmacology. 2013; 73: 216-231.

64. Copani A, Casabona G, Bruno V, et al. The metabotropic glutamate receptor mGlu5 controls the onset of developmental apoptosis in cultured cerebellar neurons. Eur J Neurosci. 1998; 10: 2173-2184.

65. Ulus IH, Wurtman RJ. Metabotropic glutamate receptor agonists increase release of soluble amyloid precursor protein derivatives from rat brain cortical and hippocampal slices. J Pharmacol Exp Ther. 1997; 281: 149-154.

66. Phillips JM, Lam HA, Ackerson LC, and Maidment NT. Blockade of mGluR5 glutamate receptors in the subthalamic nucleus ameliorates motor asymmetry in an animal model of Parkinson's disease. Eur J Neurosci. 2006; 23: 151-160.

67. Ambrosi G, Armentero MT, Levandis G, Bramanti P, Nappi G, and Blandini F. Effects of early and delayed treatment with an mGluR5 antagonist on motor impairment, nigrostriatal damage and neuroinflammation in a rodent model of Parkinson's disease. Brain Res Bull. 2010; 82: 29-38.

68. Liu F, Gong X, Zhang G, Marquis K, Reinhart P, and Andree TH. The inhibition of glycogen synthase kinase $3 \beta$ by a metabotropic glutamate receptor 5 mediated pathway confers neuroprotection to A $\beta$ peptides. J Neurochem. 2005; 95: 1363-1372.

69. Xiong N, Long X, Xiong J, et al. Mitochondrial complex I inhibitor rotenone-induced toxicity and its potential mechanisms in Parkinson's disease models. Critical Reviews in Toxicology, 2012; 42: 613-632.

70. Rangel-Barajas C, Coronel I, and Florán B. Dopamine Receptors and Neurodegeneration. Aging Dis. 2015; 6: 349-368.

71. Cabello N, Gandia J, Bertarelli DC, et al. Metabotropic glutamate type 5, dopamine $\mathrm{D} 2$ and adenosine $\mathrm{A}_{2 \mathrm{~A}}$ receptors form higherorder oligomers in living cells. J Neurochem. 2009; 109: 14971507.

Publisher's Note Springer Nature remains neutral with regard to jurisdictional claims in published maps and institutional affiliations. 